\title{
Knockdown hsa_circ_0063526 inhibits endometriosis progression via regulating the miR-141-5p / EMT axis and downregulating estrogen receptors
}

\author{
Zhangming Wei ${ }^{1,2,3,}{ }^{*}, \mathrm{Yi} \mathrm{Hu}^{4,{ }^{*}}$, Xiang $\mathrm{He}^{1,2,3,{ }^{*}}$, Mengmeng Zhang ${ }^{3}$, Xinyue Zhang ${ }^{3}$, Yali Wang ${ }^{5}$, \\ Xiaoling Fang ${ }^{3}$, Liping $\mathrm{Li}^{1,2}$ \\ ${ }^{1}$ Department of Obstetrics, The Second Clinical Medical College, Jinan University, Shenzhen People's Hospital, \\ Shenzhen 518020, Guangdong, China \\ ${ }^{2}$ The First Affiliated Hospital, Jinan University, Guangzhou 510000, Guangdong, China \\ ${ }^{3}$ Department of Obstetrics and Gynecology, The Second Xiangya Hospital, Central South University, Changsha \\ 410000, Hunan, P.R. China \\ ${ }^{4}$ The First Affiliated Hospital, Department of Obstetrics and Gynaecology, Hengyang Medical School, University of \\ South China, Hengyang 421001, Hunan, China \\ ${ }^{5}$ Xiangya Stomatological Hospital, Central South University, Changsha 410008, Hunan, China \\ *Equal contribution
}

Correspondence to: Yali Wang, Xiaoling Fang, Liping Li; email: 9320016@csu.edu.cn; fxlfxl0510@163.com, https://orcid.org/0000-0002-1022-664X; doctorlipingli@foxmail.com, https://orcid.org/0000-0003-3884-5095

Keywords: endometriosis, hsa_circ_0063526, EMT, circRNA, epigenetics

Received: March 22, $2021 \quad$ Accepted: December 8, $2021 \quad$ Published: December 30, 2021

Copyright: (C) 2021 Wei et al. This is an open access article distributed under the terms of the Creative Commons Attribution License (CC BY 3.0), which permits unrestricted use, distribution, and reproduction in any medium, provided the original author and source are credited.

\begin{abstract}
Endometriosis can cause severe social burdens. Abnormal circular RNA levels have been found to lead to changes of related gene expression, thereby mediating the occurrence and development of a series of diseases, including endometriosis. The role of circRNA in endometriosis is still in its infancy. This study will explore the role of circRNA hsa_circ_0063526 with microRNA-141-5p in the development of endometriosis. The expression levels of genes were detected by RT-qPCR. Transwell, wound-healing, and EdU assays were performed on the End1 / E6E7 cell line from the endometriosis patient. PCR and immunohistochemistry were used to detect the expression of candidate regulatory genes in ectopic lesions in an endometriosis mice model. The expression level of hsa_circ_0063526 in ectopic tissue of endometriosis patients was significantly higher than control $(P<0.05)$, The expression levels of hsa_circ_0063526 and miRNA-141-5P in ectopic tissue of endometriosis were negatively correlated $(P<0.05)$. Knockdown of hsa_circ_0063526 inhibited the invasion, migration, and proliferation ability of End1 / E6E7 cell; the inhibition of microRNA-141-5p rescued this inhibition $(P<0.05)$. In vivo experiments showed that miR-141-5p and si-hsa_circ_0063526 treatment reduced lesion size and regulated endometriosis genes. Our data suggest that hsa_circ_0063526 and miR-141-5p are possible biomarkers and therapeutic targets for endometriosis.
\end{abstract}

\section{INTRODUCTION}

Endometriosis is a common estrogen-dependent chronic disease affecting about $10 \%$ of childbearing-aged women [1]. The disease causes infertility and further malignant transformation, and $20-50 \%$ of infertile patients had endometriosis [2]. The etiology and pathogenesis of endometriosis are not clear. Sampson [3] first proposed the theory of menstrual blood reflux: the endometrial glandular epithelium and mesenchymal cells flow back into the fallopian tube and the abdominal cavity with menstrual blood, and implant in the bottom of abdominal organs such as the ovaries or pelvic peritoneum [4]. The cells continue to grow there, creating endometriosis [5]. 
Although endometriosis has benign histomorphology, its clinical behaviors show similar malignant tumors' invasion and resistance to apoptosis [5]. Recently, the pathogenesis of endometriosis has been increasingly attributed to a variety of genes and factors closely related to genetics [6]. Current treatment for endometriosis has many side effects, including stopping the menstrual cycle [7-10]. Because of this, new diagnostic indicators and non-hormonal therapy for endometriosis are urgently needed.

MicroRNAs (miRNA) are highly conserved and important post-transcriptional regulators [11]. At present, studies on the interaction between miRNA and endometriosis are gradually attracting attention. Many studies have regarded miRNA as a potential new biomarker for endometriosis [12-15].

Previous studies from our group used miRNA Solexa sequencing and RT-qPCR verification to test the serum samples of endometriosis patients in stage I and II [1619]; the results showed that the expression level of miR141 was lower than control [20]. Other studies have also demonstrated the down-regulation of the mir-200 family (including miR-141-5p) in plasma of endometriosis patients [20].

Several studies have explored the use of miR-141-5p to treat a range of diseases. For example, Kim et al. previously synthesized miR-141-5p hydrogel that demonstrated compelling results in liver cancer mice [21]. Rekker et al. reported that the expression of circulating microRNA-200s family, including miR-141$5 \mathrm{p}$, in the serum of endometriosis patients was lower than control [14]. Liang et al. used miR-200c to suppress endometriosis in vitro and in vivo [22]. It has also been reported that the reduction of miR-141-5p in endometriosis tissue promotes the development of endometriosis mainly by regulating the epithelialmesenchymal transformation (EMT) [23].

CircRNAs exist in eukaryotic cells as covalently closed rings, without 5 'or 3 ' polarity or polyadenylate, and are more conserved and stable than linear RNAs. [24] CircRNAs were previously thought to be a rare type of RNA, simply "waste products" from the process of RNA cutting [25]. However, increasing evidence has shown that circRNAs are involved in the proliferation, invasion, and metastasis of various diseases [26-29]. CircRNAs regulate gene expression levels mainly by binding to proteins or sponging microRNAs [30]. These molecular interactions will open up new prospects for diagnosing and treating endometriosis [23, 29, 31]. However, the pattern and potential role of circRNAs in the tissue of patients with endometriosis have not been elucidated.
Our previous study tested microarray circRNA expression and performed RT-qPCR verification in four endometriosis tissue samples compared to four controls. We found that the expression level of hsa circ 0063526 (circ-RanGAP1) was higher in the endometriosis group, and bioinformatics analysis revealed that circ-RanGAP1 and miRNA-141-5p have complementary binding sites [32]. We hypothesized that circRNAs might be involved in the development of endometriosis. Here, we aim to explore the correlation between hsa_circ_0063526 with miR-141$5 \mathrm{p}$ and endometriosis (Figure 1 ).

\section{RESULTS}

\section{Comparison of relative expression of hsa_circ_0063526 between endometriosis patients and the control group}

RT-qPCR was used to analyze the expression level of hsa_circ_0063526 in the endometriosis group compared to the control group. The results are shown in Figure 2. Compared with the control group, the level of hsa_circ 0063526 was significantly higher in the endometriosis group; mean \pm SEM was $0.686 \pm 0.174$ $(\mathrm{P}<0.005)$.

\section{Bioinformatics analysis and luciferase assay with hsa_circ_0063526 and miRNA-141-5p}

We used bioinformatics analysis (starBase, www.star Base.sysu.edu.cn, RNAhybrid software) to predict the downstream target miRNA to hsa_circ_0063526 previously. We performed a double luciferase reporter assay on the binding site (Figure 2B, 2C). The double luciferase assay showed that the co-transfection of hsa_circ_0063526 wild-type and the miR-141-5p mimic significantly reduced luciferase activity compared with the control group. Compared with the co-transfection of hsa_circ_0063526 mutant and miR-141-5p mimic, the reporter gene expression was rescued, indicating that miR-141-5p mimic could bind to hsa_circ_0063526.

\section{Correlation between miR-141-5p and hsa_circ_0063526 expression}

RT-qPCR was used to determine the relative expression of miR-141-5p between endometriosis patients and the control group (Figure 3A). We found a significant difference of means \pm SEM that was $0.600 \pm 0.084$ $(\mathrm{P}<0.01)$. Pearson's correlation analysis of the expression levels of hsa_circ_0063526 and miR-141-5p in the lesion tissue of endometriosis showed a negative correlation $(\mathrm{r}=$ $-0.427, \quad \mathrm{p}<0.05$, Figure 3B). Pearson's correlation analysis further indicated this negative interaction between hsa_circ_0063526 and miR-141-5p. 
Due to the overexpression of hsa circ 0063526, we constructed different siRNAs to knock down hsa circ 0063526. Three fluorescent small interfering RNAs (siRNAs) were designed and synthesized. Fluorescent images showed successful transfection of siRNA into End1/E6E7 cells, and results showed that interference sequence three significantly reduced the expression of hsa_circ_0063526 compared to the other two (Supplementary Figure 1).

After transfection with si-hsa_circ 0063526, the expression level of hsa_circ_0063526 in End1/E6E7 cells in the si-hsa_circ_0063526 group was significantly decreased $(\mathrm{P}<0.05$, Figure $3 \mathrm{C})$, and the expression level of miR-141-5p was significantly increased $(\mathrm{P}<0.05$, Figure 3D), compared with that in the blank group and the si-NC (Negative control) group. The results showed that hsa_circ_0063526 negatively regulated the expression level of miR-141$5 p$ in End1/E6E7 cells.

\section{Down-regulation of hsa_circ_0063526 can inhibit the proliferation, invasion, and migration of End1 / E6E7 cells}

Migration, invasion, and proliferation of ectopic endometrial cells are essential pathological processes for the development of endometriosis. Therefore, 72 hours after transfection of si-hsa_circ_0063526, this study further explored the changes of cell migration and invasion and cell proliferation ability after downregulation of hsa_circ_0063526. After End1 / E6E7 cells were transfected with hsa_circ_0063526 siRNA, fewer cells entered the lower chamber than the control group, demonstrating that cell invasion ability decreased (Figure 4). The ability of cell migration was also tested by a wound healing experiment; after End1 / E6E7 cells were transfected hsa_circ_0063526 siRNA, the width of the cell scratch was broader than that of the control group, demonstrating decreased cell migration ability (Figure 5).
A

Case group VS Control group sequencing

whole transcriptomeseq / CircRNA-seq

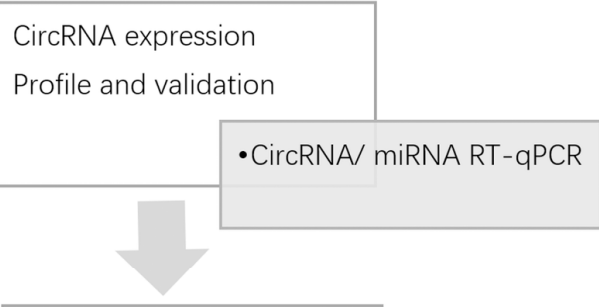

Specific CircRNA function assays

- Bioinformatics prediction - In vitro / in vivo validation
B
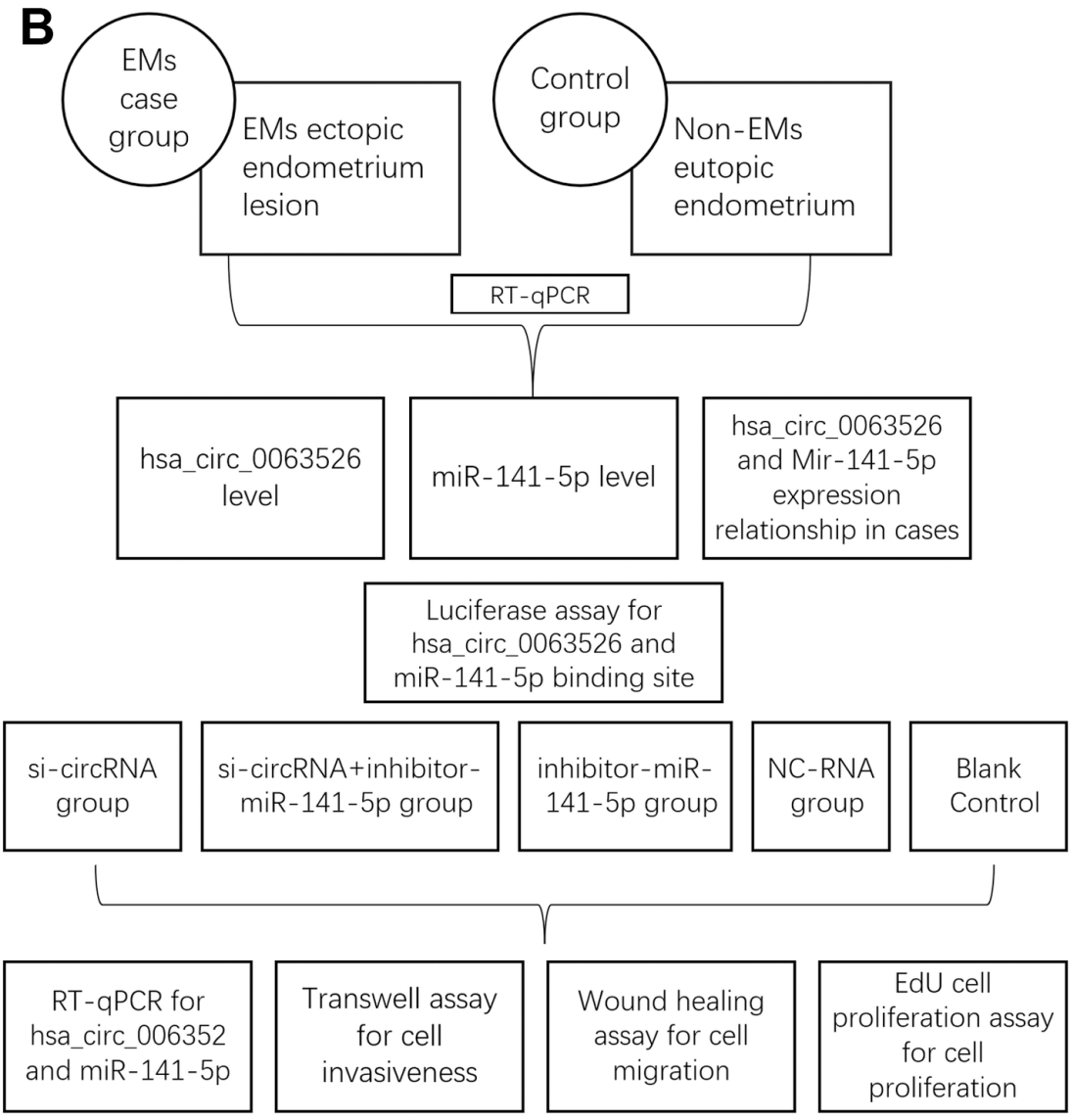

In vivo experimental hsa_circ_0063526 treatment of endometriosis mice
In vivo experimental miR-141-5p treatment of endometriosis mice

Figure 1. Study design. (A) Whole research design. sequencing, profile validation and function bioinformatics part has been done and published in our previous articles; (B) Experimental design in this study. 
Further, the effect of down-regulation of hsa_circ_0063526 on cell proliferation was detected using the $\mathrm{EdU}$ cell proliferation assay. By $72 \mathrm{~h}$ after siRNA transfection, fewer End1/E6E7 cells were in the mitotic stage after the downregulation of hsa circ 0063526, and the proliferation capacity of the cells was decreased after siRNA transfection compared to control. Difference between means $\pm \mathrm{SEM}=32.38 \pm 6.639(\mathrm{P}<0.05$, Figure 6$)$. PCR and ELISA results showed that the expression of Ecadherin mRNA, an important epithelial marker of EMT, was upregulated $(\mathrm{P}<0.05$, Figure 7$)$.

\section{Downregulation of miR-141-5p can rescue the proliferation, invasion, and migration of endometriosis inhibited by hsa_circ_0063526}

After cells were co-transfected with sihsa_circ_0063526+inhibitor-miR-141-5p, the proliferation, invasion, and migration of endometriosis cells were rescued $(\mathrm{P}<0.05$, Figures $4-6)$. $\mathrm{PCR}$ and ELISA results showed that the expression of E-cadherin, an essential epithelial marker of EMT, was downregulated compared with the si-hsa_circ_0063526 group $(\mathrm{P}<0.05$, Figure 7$)$.

The above experimental results showed that knockdown hsa_circ_0063526 might inhibit the development of endometriosis. Inhibition of microRNA-141-5p can rescue the inhibitory effect brought by knockdown of hsa_circ_0063526. Therefore, we hypothesize hsa_circ_0063526 promotes the development of endometriosis through inhibition of microRNA-141-5p.

\section{Comparison of miR-141-5p treatment and pathological changes}

No adverse reactions were observed in mice treated with miR-141-5p. At the end of miRNA-141-5p

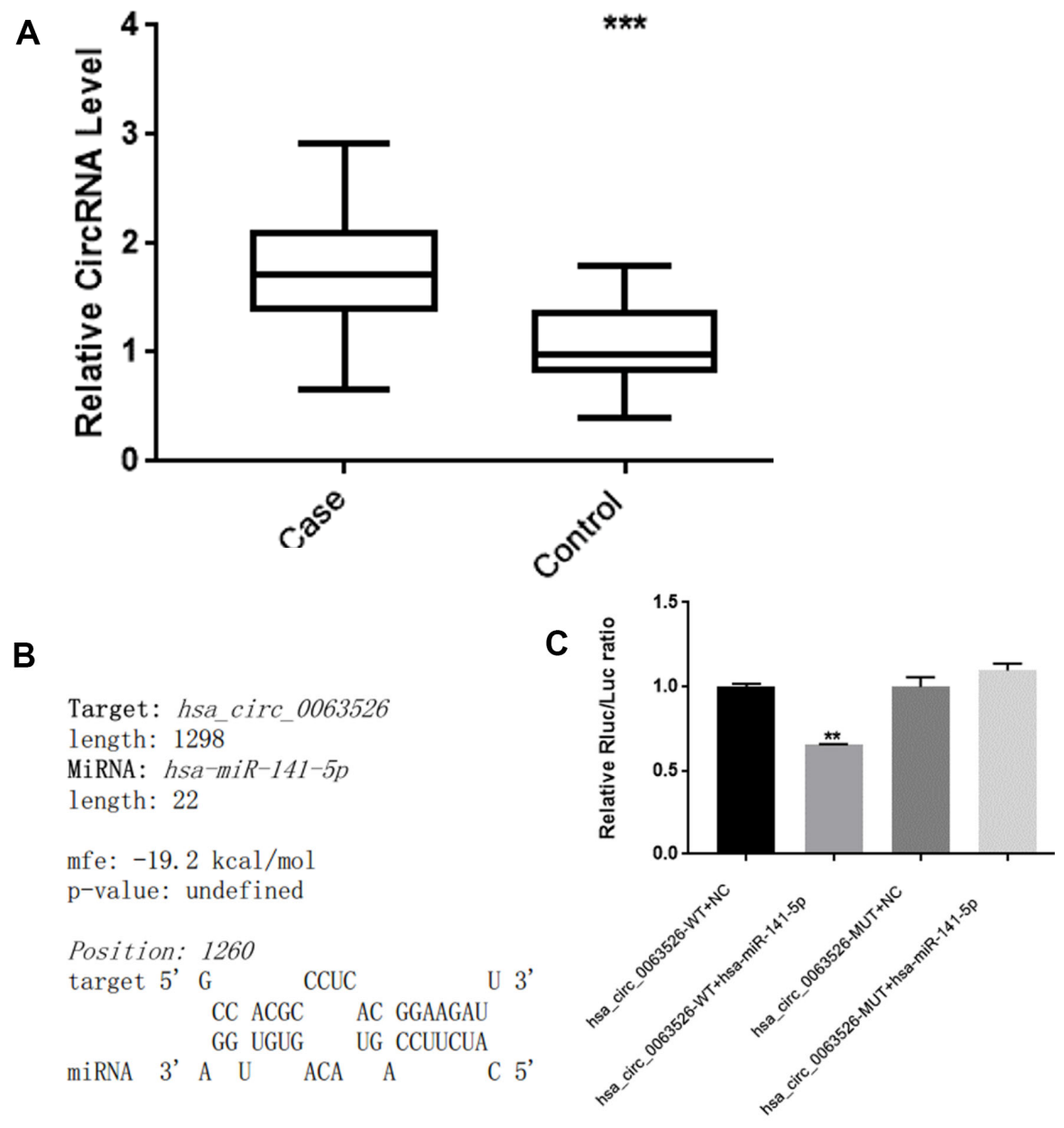

Figure 2. hsa_circ_0063526 level is higher in EMs ectopic endometrial lesions than control's eutopic endometrial tissue and hsa_circ_0063526 have binding site with miRNA-141-5p. (A) RT-qPCR analysis of the hsa_circ_0063526 level in the endometriosis group and control group by RT-qPCR. $n=41$ (*** $\mathrm{P}<0.005)$. (B) hsa_circ_0063526 complementary sequence with miR-141-5p. (C) Luciferase reporter experiment showed that miR-141-5p can bind to hsa_circ_0063526 at this site. Replicated 3 times with 3 separate samples (** $P<0.01$ ). 
treatment, mice were sacrificed by cervical dislocation and endometriosis lesions were collected and the volume of the lesions between the miR-141-5p treatment group and the control group were evaluated. All lesions were cystic and smallest in the miR-141-5p treated groups (Figure 8A-8C).
Further, the histological area of all lesions was observed under the microscope. The histological area of endometriosis in abdominal lesions of the miR-141-5p treatment group was significantly smaller than the two control groups $(\mathrm{p}<0.05$, Figure $8 \mathrm{D}-8 \mathrm{G})$.
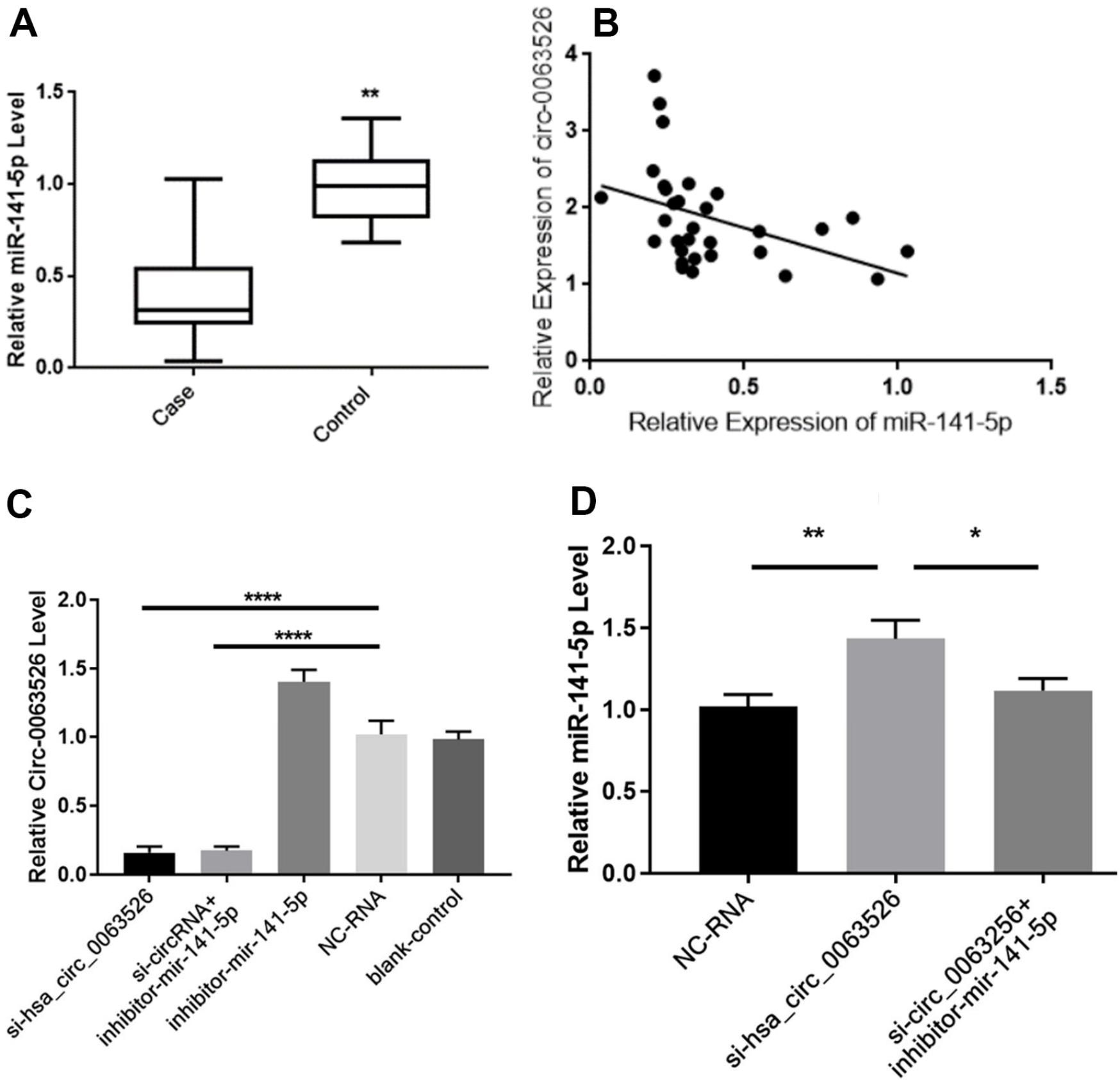

Figure 3. Hsa_circ_0063526 and miR-141-5p expression level is negative correlated. (A) Quantitative analysis of miR-141-5p expression levels in endometriosis group and control group by RT-qPCR. $n=41$ (** $P<0.01)$. (B) Pearson's correlation analysis of the correlation between hsa_circ_0063526 and miR-141-5p expression level in the endometriosis group. $n=31(P<0.05)$ (C) RT-qPCR shows the relative expression level of hsa_circ_0063526 in End1/E6E7 cells after downregulation of hsa_circ0063526 or inhibition of miR-141-5p. (**** $P<0.001)$. (D) RT-qPCR shows the relative expression level of miR-141-5p in End1/E6E7 cells after down-regulation of hsa_circ_0063526. $(*(P<0.05, * * P<0.01)$. Replicated 3 times with 3 separate samples. 


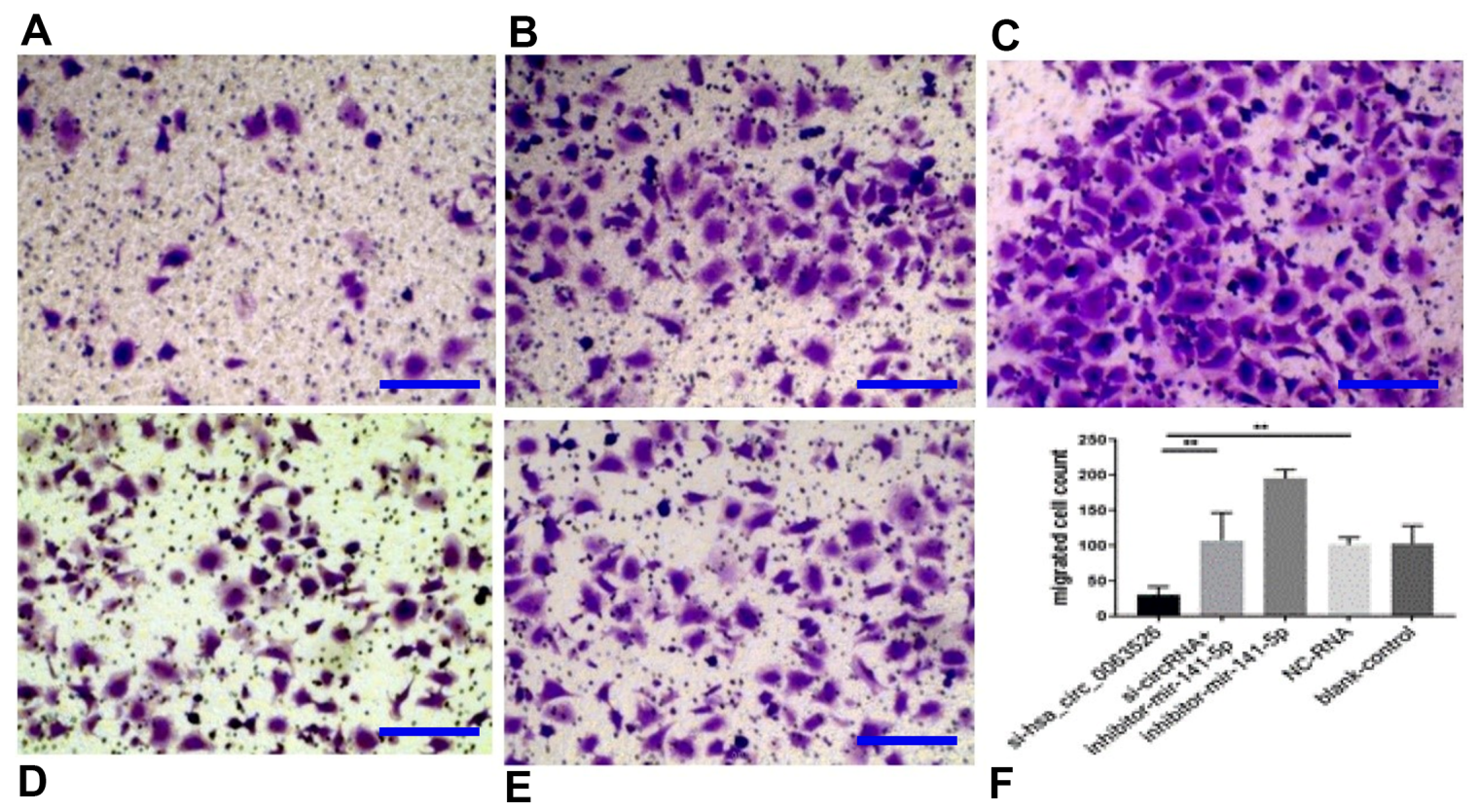

Figure 4. Transwell assay to investigate the effect of inhibition of hsa_circ_0063526 or/and miR-141-5p on cell invasion of End1/E6E7 cells. (A) si-hsa_circ_0063526 group; (B) si-hsa_circ_0063526 +inhibitor miR-141-5p group; (C) miR-141-5p Inhibitor group; (D) NC-RNA group; (E) Blank control group; (F) The number of cells comparing to the NC-RNA control group. after End1 / E6E7 cell transfected hsa_circ_0063526 siRNA, the cells entering the lower chamber through the pore was lesser compared to the control group, cell invasion ability decreased. After co-transfection with si-hsa_circ_0063526 + inhibitor miR-141-5p, the invasion ability of End1/E6E7 cells was rescued. Replicated 3 times with 3 separate samples (* $\mathrm{P}<0.05, * * \mathrm{P}<0.01)($ bar:25 $\mu \mathrm{m})$.

A

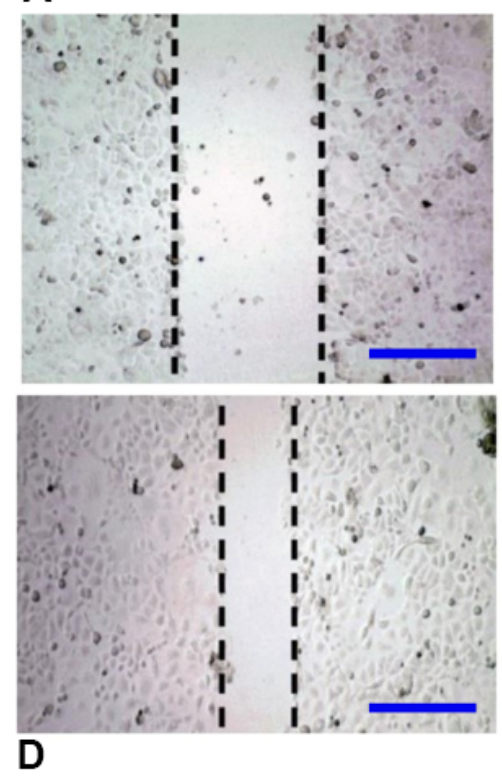

B

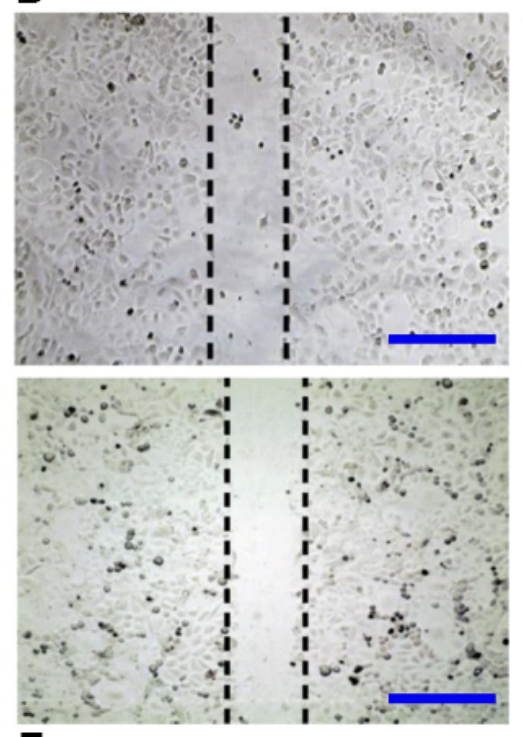

E
C

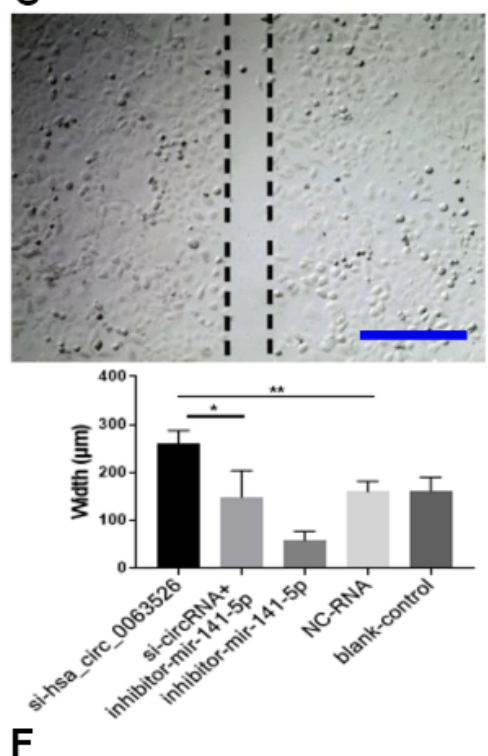

Figure 5. Wound-healing test on the effect of inhibition of hsa_circ_0063526 or/and miR-141-5p on cell migration in End1/E6E7 cells. (A) si-hsa_circ_0063526 group; (B) si-hsa_circ_0063526 +inhibitor miR-141-5p group; (C) miR-141-5p Inhibitor group; (D) NC-RNA group; (E) Blank control group; (F) The scratch width comparing to the si-NC group. After transfection with hsa_circ_0063526 siRNA in End1/E6E7 cells, the cell migration ability was decreased compared with the control group. After co-transfection with si-hsa_circ_0063526 + inhibitor miR-141-5p, the cell migration ability of End1/E6E7 cells was rescued. Replicated 3 times with 3 separate samples (* P<0.05, **

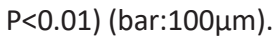


A
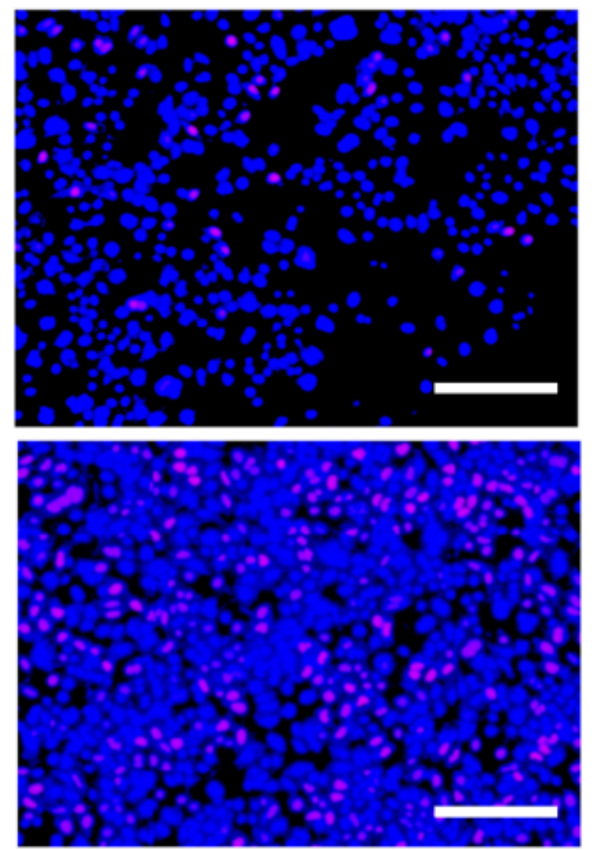

D
B
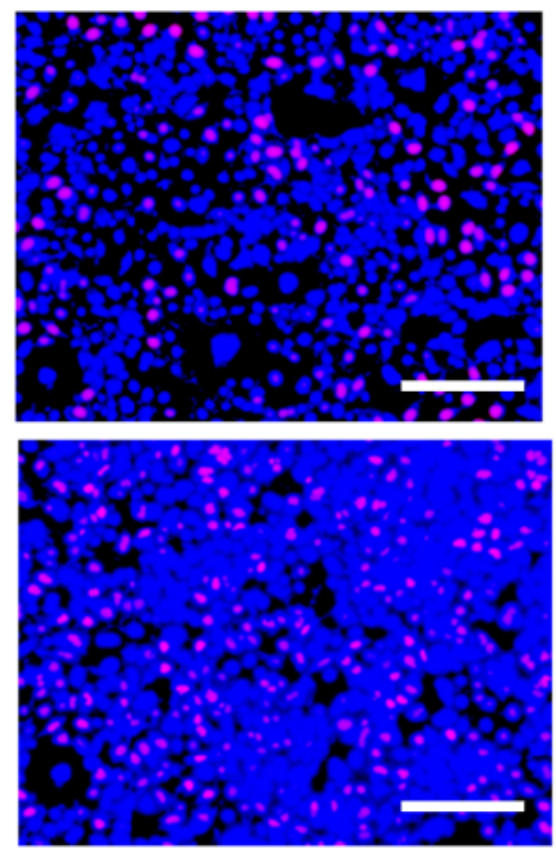

$\mathbf{E}$
C
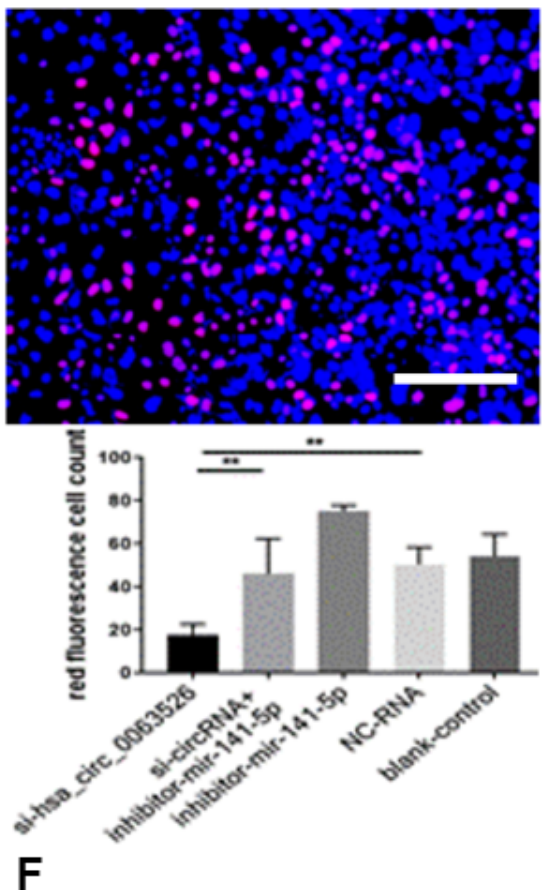

Figure 6. EdU assay was used to investigate the effect of inhibition of hsa_circ_0063526 or/and miR-141-5p on the proliferation of End1/E6E7 cells. (A) si-hsa_circ_0063526 group; (B) si-hsa_circ_0063526 +inhibitor miR-141-5p group; (C) miR-141-5p Inhibitor group; (D) NC-RNA group; (E) Blank control group; (F) the number of red fluorescent cells. After transfection with hsa_circ_0063526 siRNA in End1/E6E7 cells, the number of cells in the control group was lower, and the proliferation ability of cells in endometriosis was decreased. After co-transfection with si-hsa_circ_0063526 + inhibitor miR-141-5p, the proliferation ability of End1/E6E7 cells was rescued.

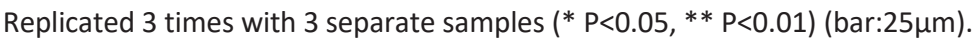

A

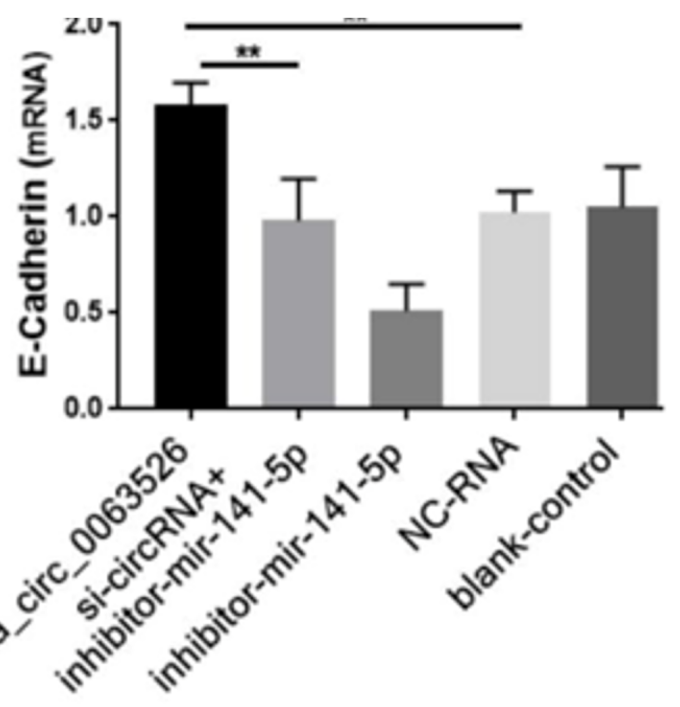

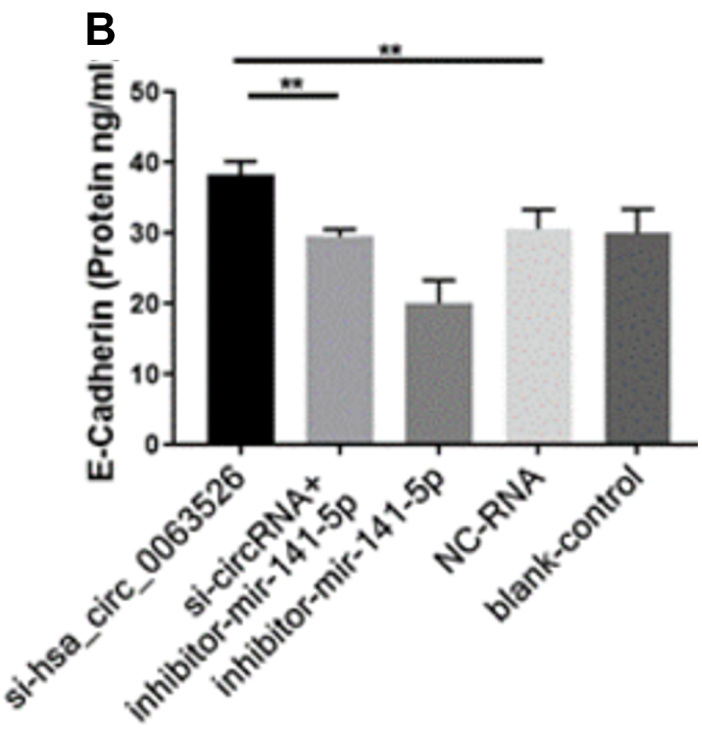

Figure 7. RT-qPCR and ELISA assay to investigate the effect of inhibition of hsa_circ_0063526 or/and miR-141-5p on the expression of E-cadherin in End1/E6E7 cells. (A) The expression level of E-cadherin mRNA (B) Expression level of E-cadherin protein. Ecadherin expression was upregulated. Expression changes were recovered after co-transfection with si-hsa_circ_0063526 + inhibitor- miR141-5p. Replicated 3 times with 3 separate samples $(* P<0.05, * * P<0.01)$. 


\section{Expression difference of endometriosis-related genes}

The differences in gene expression levels related to endometriosis were detected by RT-qPCR. The expression levels of some genes known to promote the development of endometriosis decreased in the miR141-5p group. Expression levels of N-cadherin, Vimentin, K-ras, MAPK-14, ER- $\alpha$, ER- $\beta$, and ZEB-1 were decreased in the miR-141-5p treatment group $(\mathrm{P}<0.05$, Figure 9). The decrease fold in gene
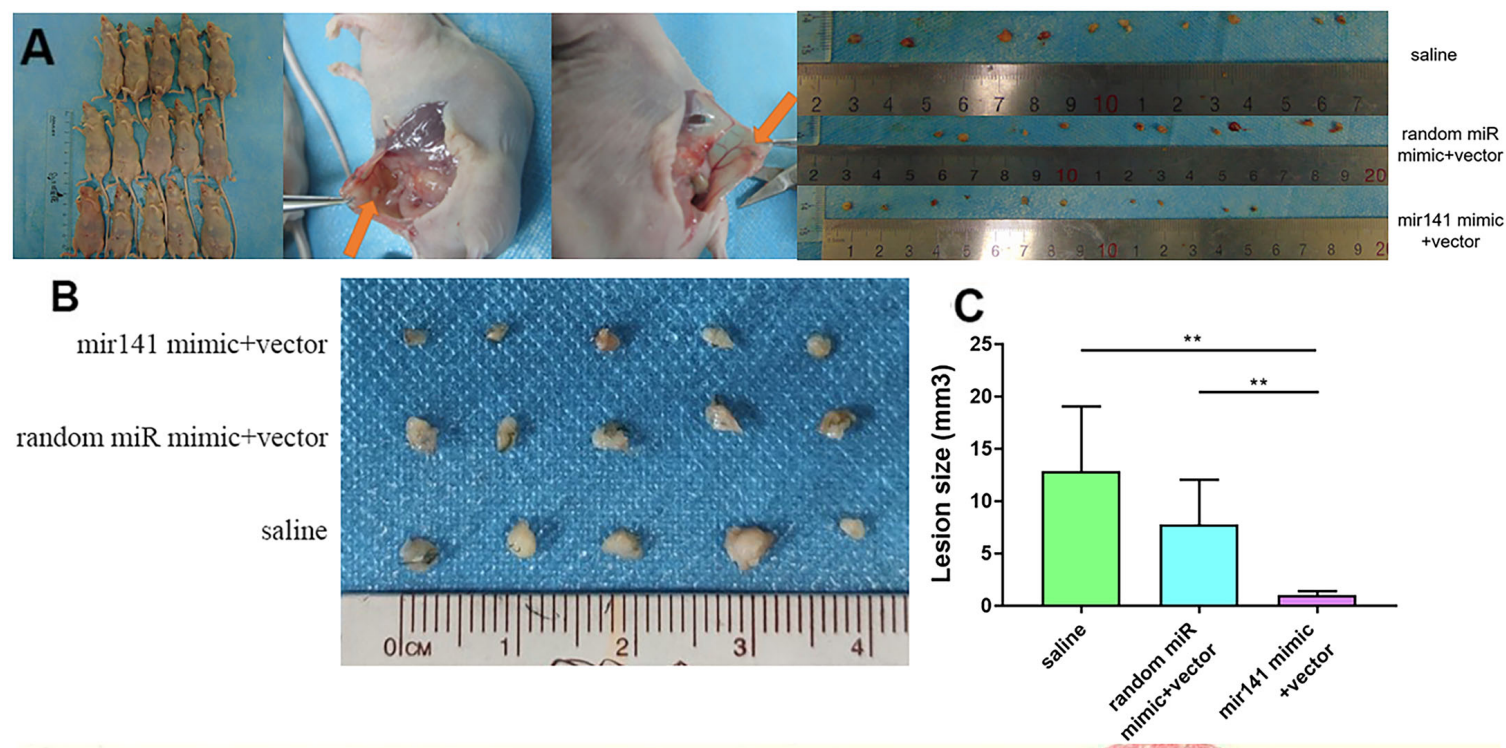

area $=4.36382 \mathrm{~mm}^{3} \quad$ mir141 mimic +vector
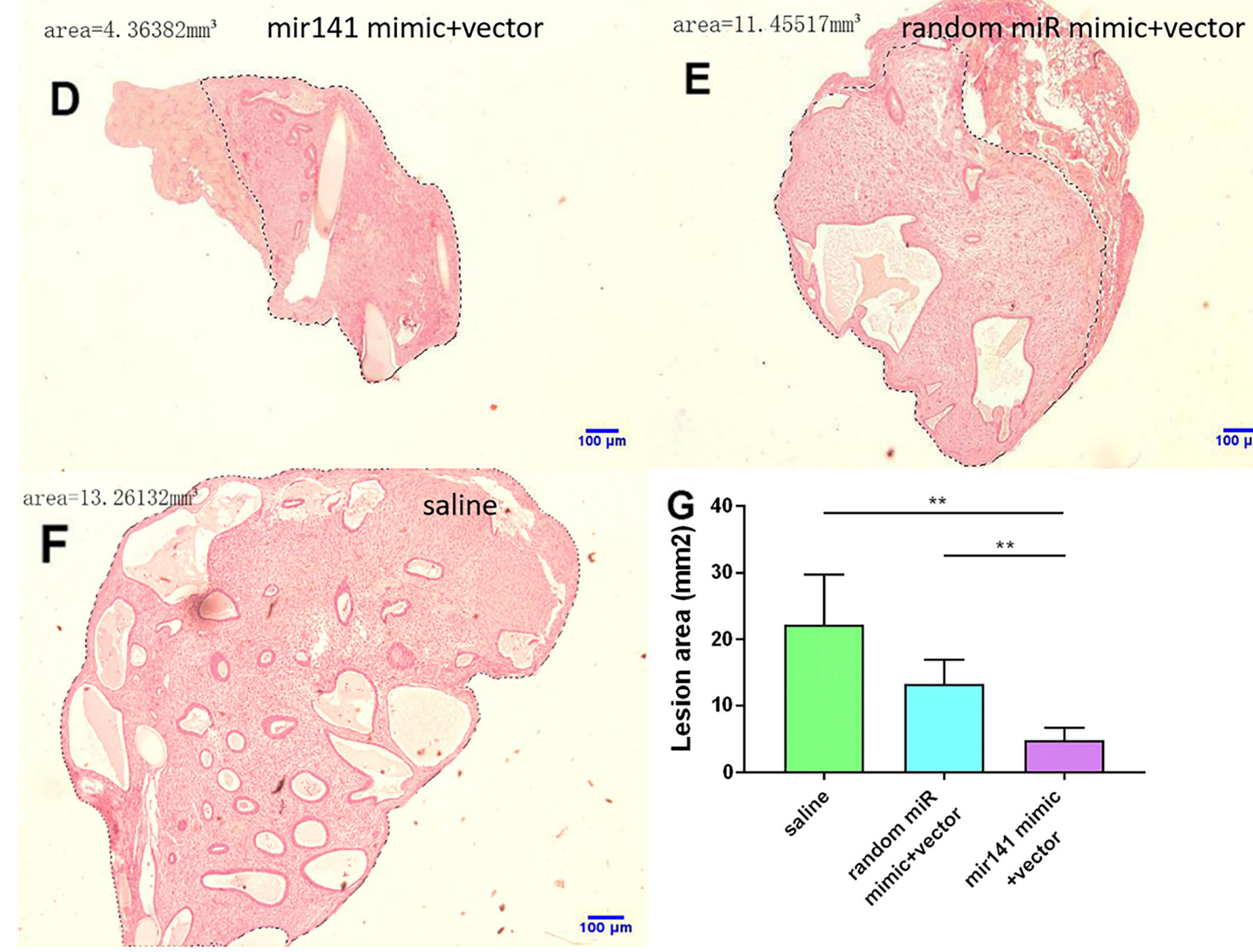

$\overline{100 \mu \mathrm{m}}$

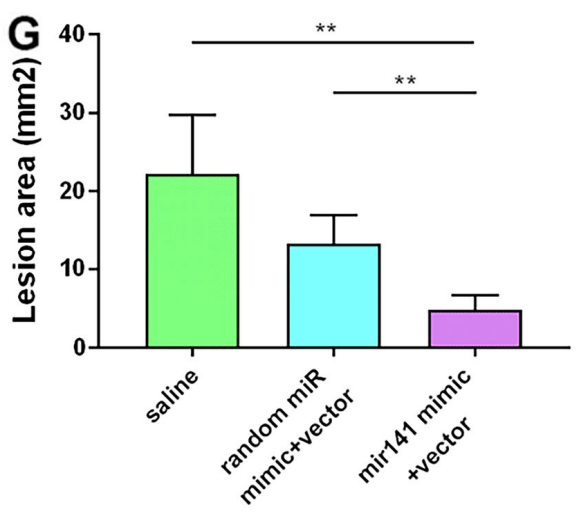

Figure 8. Collection and comparison of lesion tissues from each mouse. (A) The collection of endometriosis lesions. (B, C) Comparison of left abdominal lesion volume; (5 mice in each group). (D-G) The difference of histological area of the median section of endometriosis in mice of the upregulated group and control group under H\&E staining $(* P<0.05, * * P<0.01)$. 

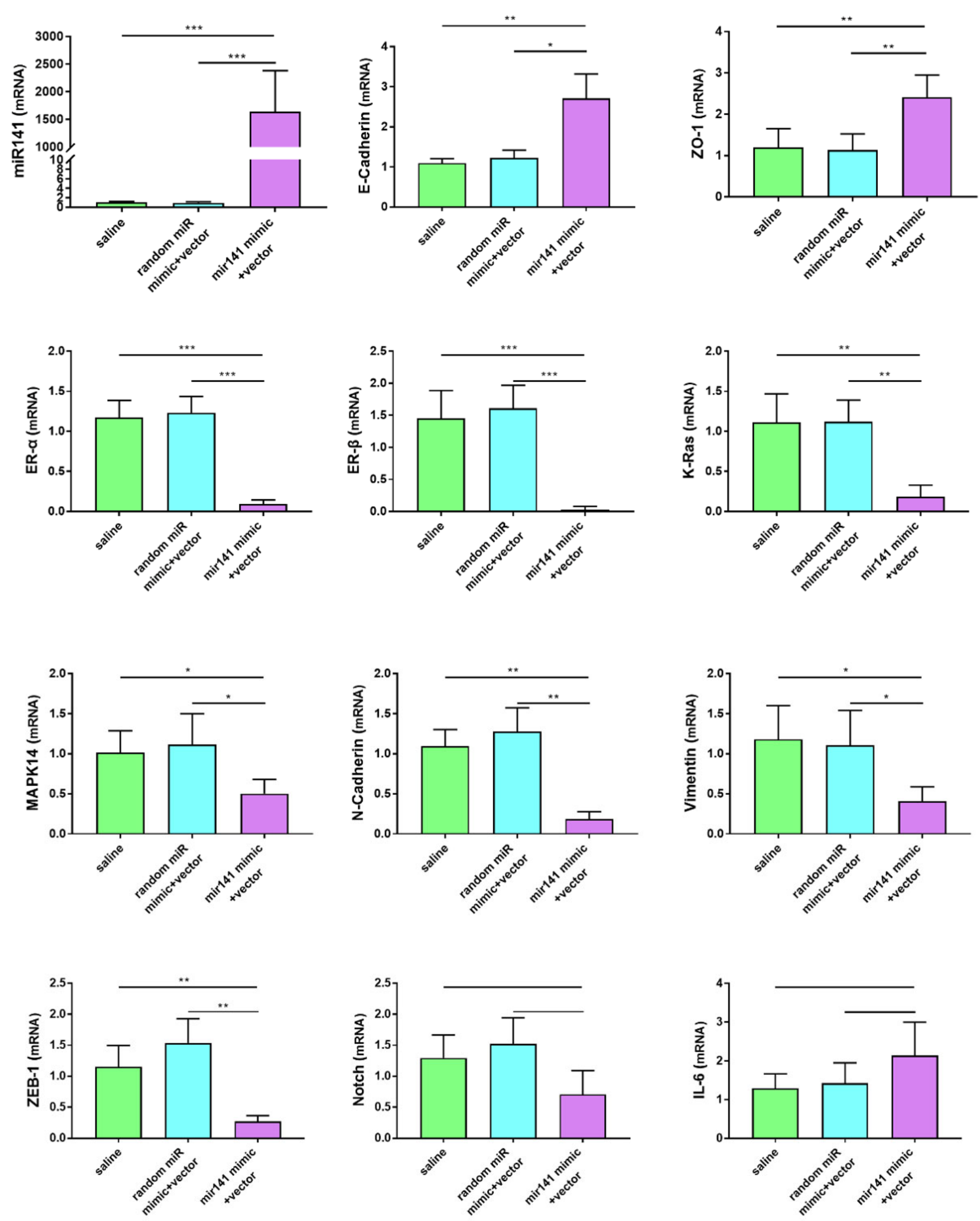

Figure 9. Effects of miR-141-5p treatment on mRNA expression of pathologically related genes in endometriosis detected by RT-qPCR. miR-141-5p treatment significantly decreased n-cadherin, Vimentin, K-ras, MAPK-14, ER- $\alpha$, ER- $\beta$, ZEB-1 expression levels, and increased E-cadherin, ZO-1 expression levels. The data were shown as average percentage \pm SEM. Replicated 3 times with 3 separate samples $\left({ }^{*} \mathrm{P}<0.05,{ }^{* *} \mathrm{P}<0.01\right)$. 
expression is 6.5-fold for K-Ras, 1.81-fold for MAPK14, 13.9-fold for ER- $\alpha$, 97.2-fold for ER- $\beta$, 4.63-fold for N-Cadherin, 2.37-fold for Vimentin, 3.98-fold for ZEB-1 in 141 treated group compared to saline group. Expression levels of miR-141, ECadherin, ZO-1 (Zona Occludens 1) were increased in the miR-141-5p treatment group $(\mathrm{P}<0.05$, Figure 9$)$. Expression levels of Notch and IL-6 were not statistically significant between treatment and control groups ( $\mathrm{P}>0.05$, Figure 9$)$.

Immunohistochemistry results showed that N-cadherin and vimentin protein levels in stromal cells decreased in the miR-141-5p treatment group (Figure 10).

\section{Comparison of si-hsa_circ_0063526 treatment and pathological changes}

No adverse reactions were observed in mice treated with si-hsa circ 0063526 agomir. At the end of si- hsa circ 0063526 agomir treatment, mice were sacrificed by cervical dislocation and endometriosis lesions were collected. We evaluated the volume of the lesions between the si-hsa circ 0063526 agomir treatment group and the control group. All lesions were cystic. Lesions in si-hsa_circ_0063526 agomir treatment groups were relatively small (Figure 11A).

Further, the histological area of all lesions was observed under the microscope, and the histological area of abdominal lesions in the miR-141-5p treatment group was significantly smaller than the two control groups ( $p$ $<0.05$, Figure 11B-11E).

\section{Endometriosis-related gene expression differences}

RT-qPCR was used to detect the gene expression differences associated with endometriosis. Compared with the control group, some genes known to promote the development of endometriosis were decreased in the

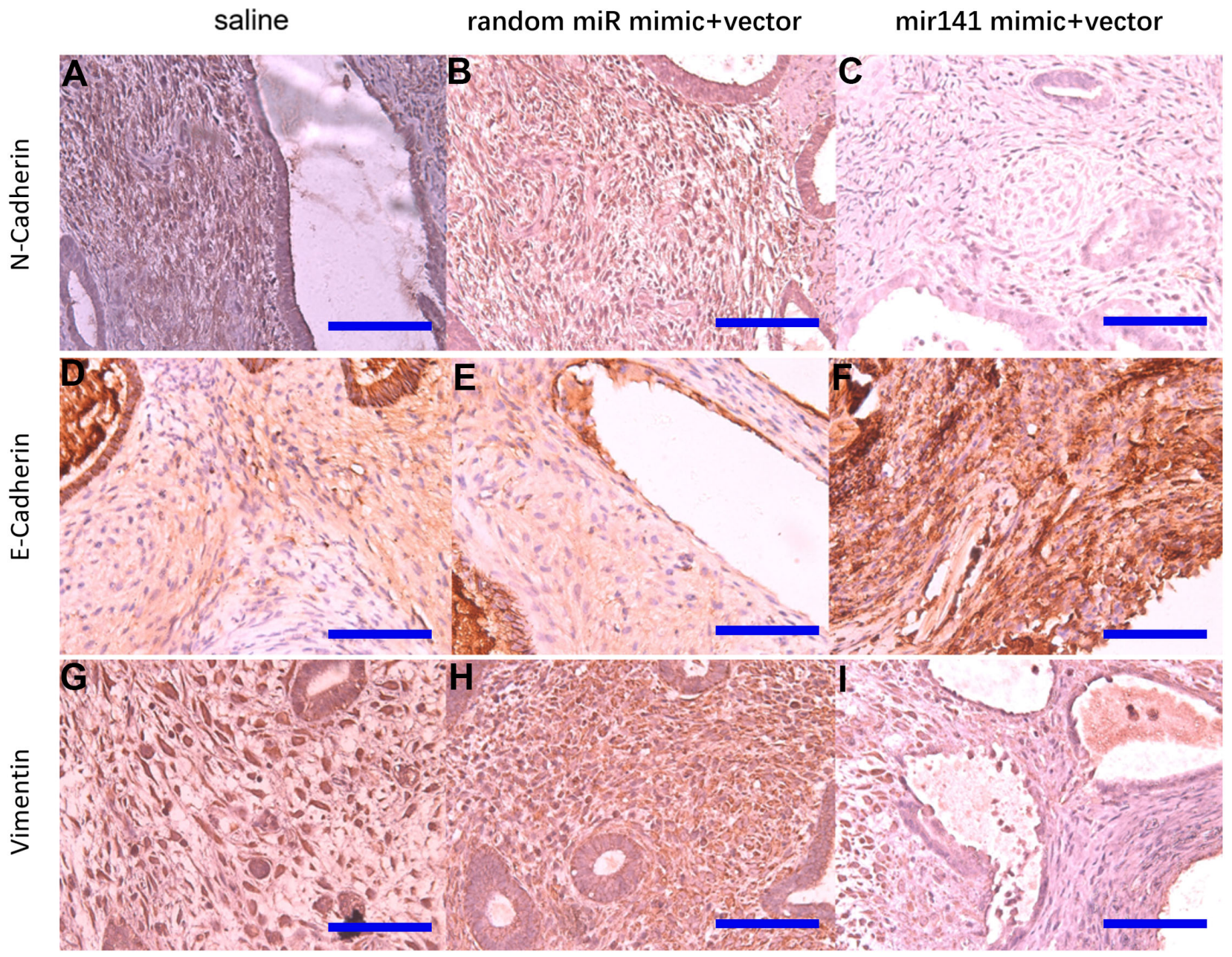

Figure 10. Representative images of $\mathrm{N}$-cadherin, E-cadherin, and Vimentin protein levels detected by immunohistochemistry. $\mathrm{N}$-cadherin protein $(\mathbf{A}-\mathbf{C})$, E-cadherin protein (D-F), Vimentin protein $(\mathbf{G}-\mathbf{I})($ bar:50 $\mu \mathrm{m})$. 
si-hsa_circ_0063526 agomir group, including MAPK14 , K-ras, N-cadherin, Vimentin, ER- $\alpha$, ER- $\beta(\mathrm{P}<$ 0.05 , Figure 12). K-ras, MAPK14, ER- $\alpha$, ER- $\beta$, and $\mathrm{N}$-cadherin decreased between 2-8 fold (Figure 12). The expression levels of E-cadherin and Zona occludens 1 (Zona Occludens 1) in the si- hsa_circ_0063526 agomir group were increased $(\mathrm{P}<0.05$, Figure 12).

Immunohistochemical results showed that E-cadherin staining intensity was significantly increased in the sihsa_circ_0063526 treatment group (Figure 13).

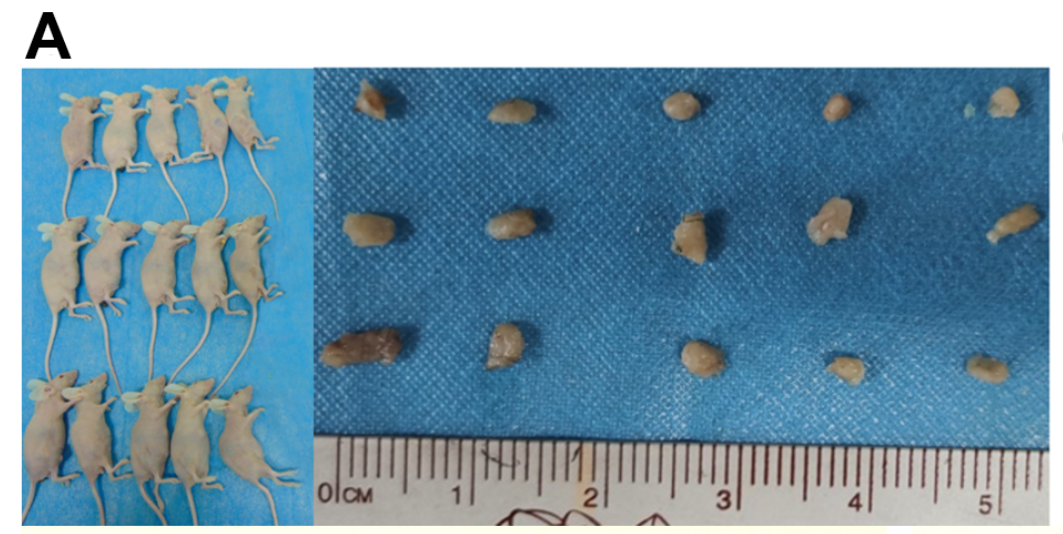

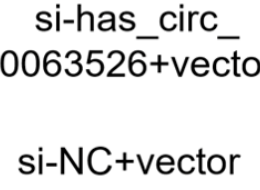

saline

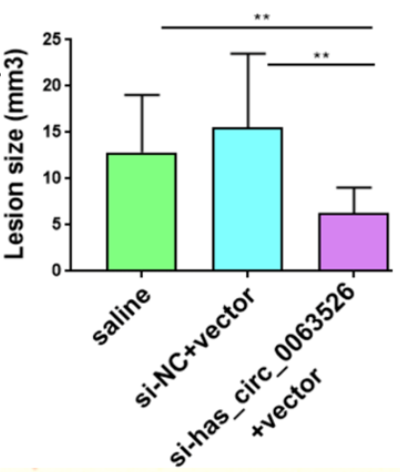

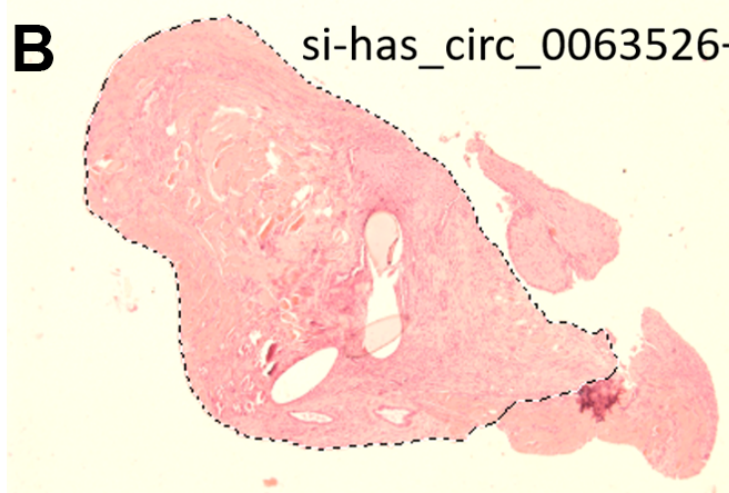

ector
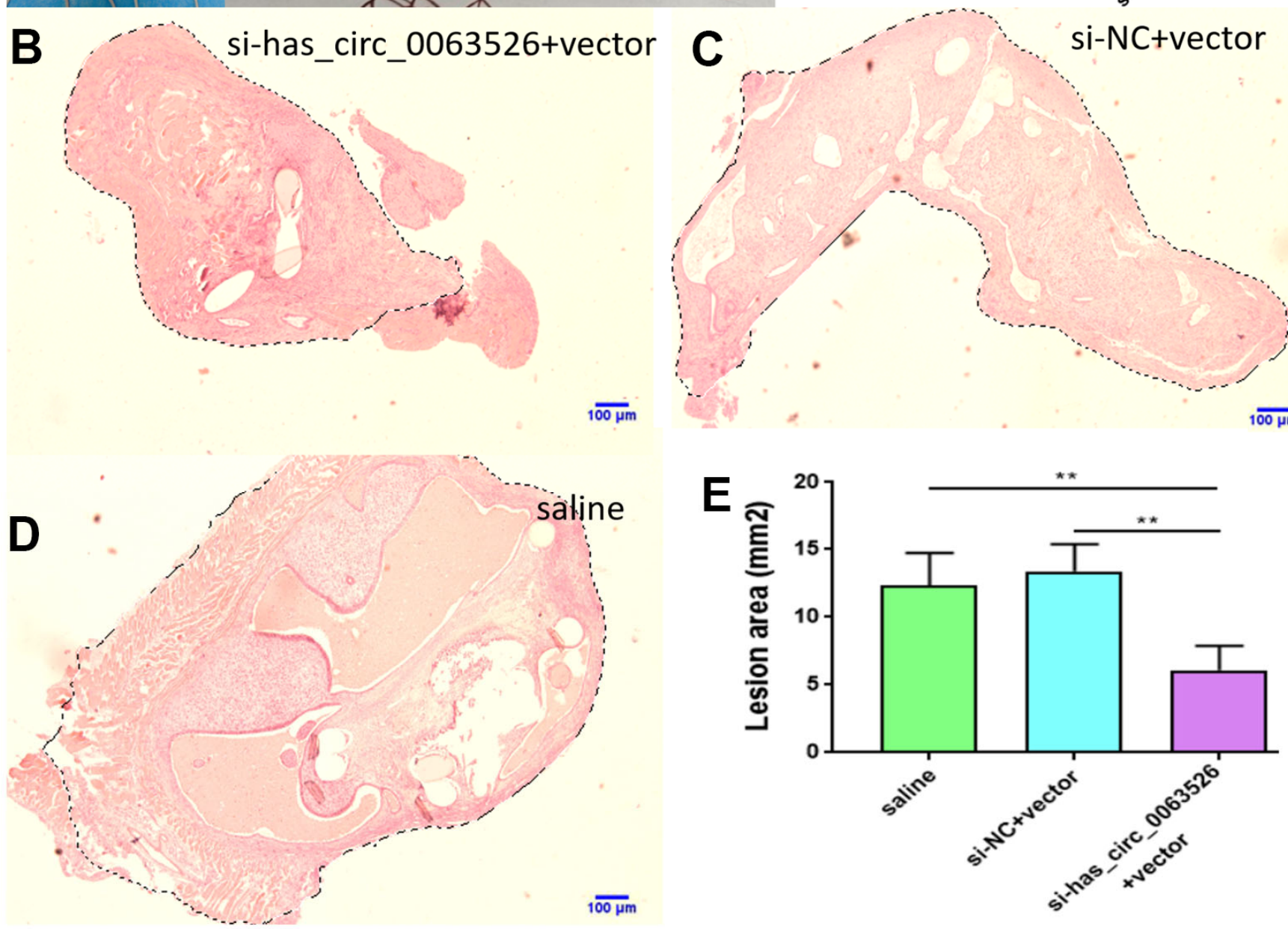

Figure 11. Tissue collection and comparison of each mouse. (A) Comparing the volume of lesions in the left abdomen; (5 mice in each group). (B-E) The difference of histological area of the median section of endometriosis in mice of the si-hsa_circ_0063526 treatment group and control group under H\&E staining. Data are expressed as mean \pm SEM, $* P<0.05$, $* * \mathrm{P}<0.01$. 


\section{DISCUSSION}

Endometriosis is a common estrogen-dependent chronic disease that affects about $10 \%$ women of childbearing age. While it is histomorphology is benign, its clinical behavior is similar malignant tumor invasion and resistance to apoptosis [6].

Searching for biomarkers for the detection and treatment of endometriosis has been an important area
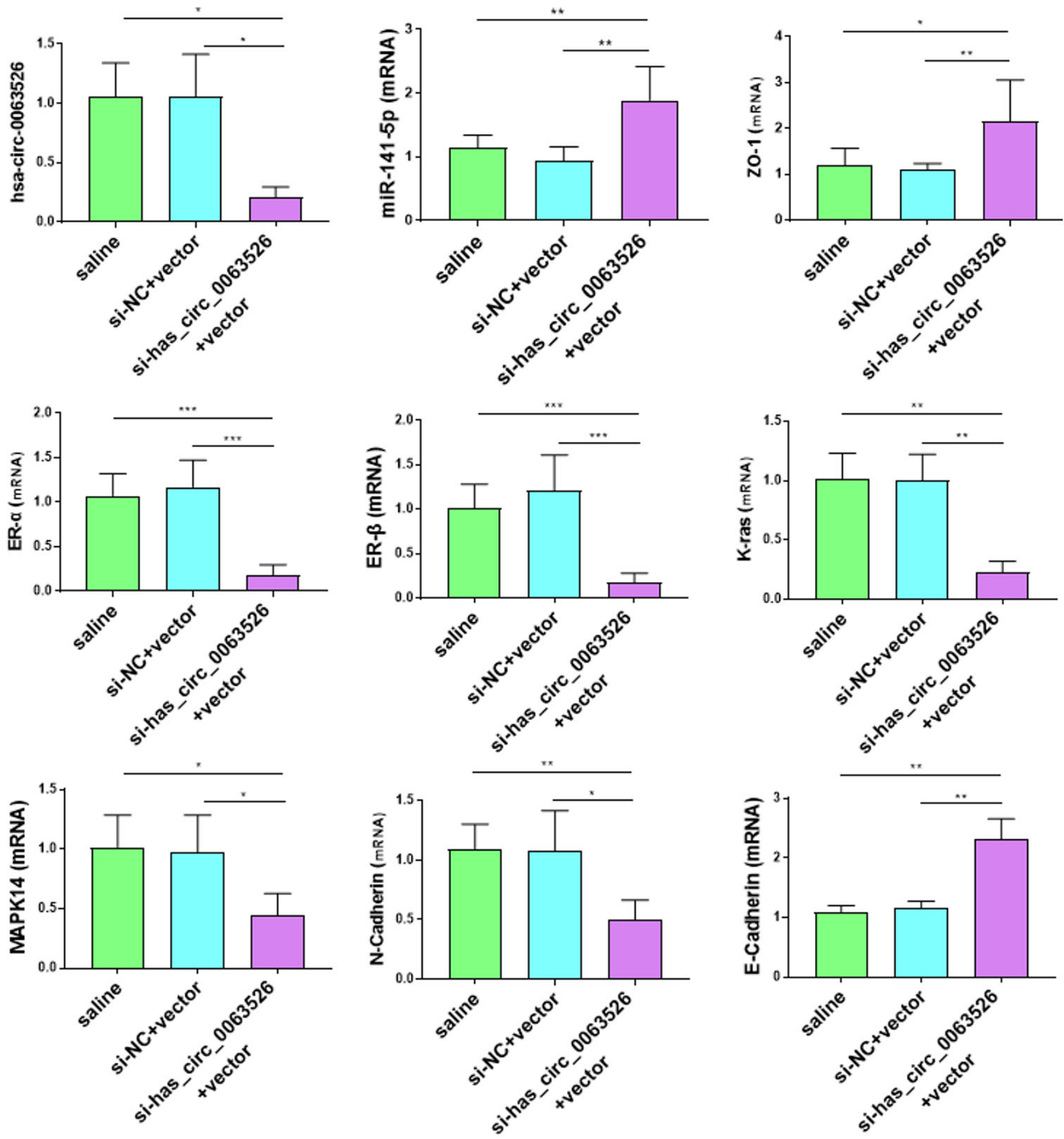

Figure 12. Effects of si-hsa_circ_0063526 treatment on mRNA expression of pathologically related genes in endometriosis detected by RT-qPCR. The expression levels of N-cadherin, K-ras, MAPK-14, ER- $\alpha$, ER- $\beta$, in the si-hsa_circ_0063526 treatment group were significantly decreased, while the expression levels of E-cadherin and ZO-1 were increased. The data were shown as average percentage \pm SEM. Replicated 3 times with 3 separate samples $\left({ }^{*} \mathrm{P}<0.05, * * \mathrm{P}<0.01\right)$. 
of research. The study of circRNA in endometriosis is still in its infancy, and its pattern and potential role in endometriosis tissues have not been elucidated [23, 33, 34]. Most of the studies on circRNA are preliminary, and more in-depth studies are needed to provide a sufficient basis for circRNA to be a diagnostic marker or therapeutic target for endometriosis [26, 29, 30, 35].

We verified that hsa_circ_0063526 was increased in endometriosis lesions, and the invasion, migration, and proliferation of End1/E6E7 cells were decreased after knockdown of hsa_circ_0063526. Bioinformatics has also shown that hsa_circ_0063526 may bind miR-141$5 \mathrm{p}$ as a target, and the dual-luciferase reporter assay further suggested that miR-141-5p can be combined with hsa_circ 0063526. In endometriosis lesions, the expression of both has a negative correlation, and our preliminary studies suggest that miR-141-5p regulates the development of endometriosis. Together, these data suggest that hsa_circ_0063526 may play a regulatory role in the occurrence and development of endometriosis through miR-141-5p.

Endometriosis is known for its cancer-like behavior [36]. It is characterized by aggressive invasion growth and a high risk of recurrence, similar to a malignant tumor $[37,38]$, therefore having similarities in pathogenesis. EMT endows cells with the ability to invade and metastasize, including reducing apoptosis, suppressing the immune response, and obtaining stem cell characteristics [39]. It not only plays a crucial role in embryonic development but is also involved in tissue healing, organ fibrosis, and the development of cancer [40]. Simpson's theory of menstrual blood reflux suggests that EMT is one of the drivers of endometriosis pathogenesis. After EMT, small mesothelial cells no longer provide a lamellar protective barrier between the basal layer and the lacunae. Because there is no protective barrier, endometrial cells tend to adhere to the peritoneal matrix, resulting in endometriosis. The expression of epithelial markers in endometriosis is down-regulated, and the expression of mesenchymal markers is upregulated [41]. EMT can resist apoptosis and assist the metastasis of ectopic lesions [42]. Second, the endometrium is formed by the transformation of the stromal cells during the development of the urogenital system of the embryo. Due to the retention of some stromal origin imprints, endometrial epithelial cells tended to revert to the original stromal state through EMT [43, 44]. We found that the EMT activity in the si-hsa_circ_0063526 group was decreased, and the expression level of E-cadherin was increased.

The Ras/MAPK signaling pathway is essential in embryo development, differentiation, proliferation, cell death, and other critical cellular processes [45]. K-ras activation is a classical method to establish a mouse model of spontaneous endometriosis and is upregulated $[36,46]$. Here, we found that the expression level of $\mathrm{K}$ ras was lower in the miR-141-5p and sihsa_circ_0063526 transfected groups compared to the control.

Compared with linear RNA, circRNAs have a more stable structure and can resist the degradation of multiple RNA enzymes. Therefore, circRNAs could be a diagnostic and therapeutic target for endometriosis $[47,48]$. Currently, no studies have been reported on the role of hsa_circ_0063526 in endometriosis, but other circRNA studies have shown that circRNA has potential value in prognosis prediction or early diagnosis of endometriosis.

Endometriosis is considered an estrogen-dependent disease, and ER- $\alpha \& \beta$ have essential roles in

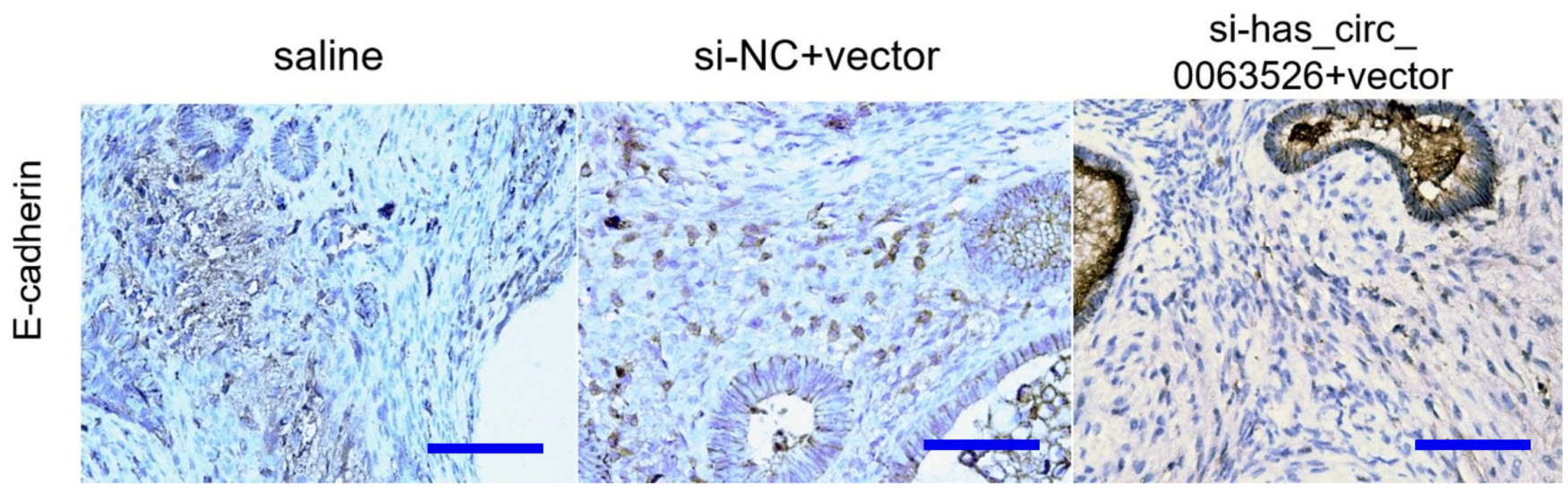

Figure 13. Representative images of E-cadherin protein levels detected by immunohistochemistry (bar:50 $\mu \mathrm{m})$. 
endometriosis [42]. ER- $\alpha \& \beta$ expression was significantly inhibited with miR-141-5p and sihsa_circ 0063526, suggesting that sihsa_circ_0063526/miR-141-5p pathway blocks estrogen stimulation in the treatment. These data reveal that miR-141-5p and si-hsa_circ_0063526 therapy may specifically block the sex hormone pathway in patients with endometriosis without systemic side effects of estrogen deficiency.

In this study, miR-141-5p and si-hsa_circ_0063526 were used for local therapy. After systematic administration, oligonucleotides have difficulty reaching the desired tissue because they are degraded by the liver and removed from the blood [49]. Therefore, many vectors have been used to improve the stability of oligonucleotides; however, these drugs are difficult to achieve targeted drug delivery [21]. We posit that miR141-5p and si-hsa_circ_0063526 have therapeutic effects as an intraperitoneal injection [50]. However, since animal models cannot wholly simulate the patient's condition, the model could not reflect the role of inflammation, angiogenesis, and other activities in endometriosis, and the side effects of the therapy could not be observed as thoroughly as in the patient. Before being used in humans, dose-response and safety studies must be conducted.

In summary, miR-141-5p and si-hsa_circ_0063526 therapy reduce endometriosis development. The pleiotropic nature of miR-141-5p and sihsa_circ_0063526 therapy suggests that multiple complementary mechanisms play a role in endometriosis. These effects suggest that endometriosis may be treated more comprehensively without the systemic side effects of current drugs, but further doseresponse and safety studies are needed before they can be used in humans.

\section{MATERIALS AND METHODS}

\section{Materials}

\section{Clinical specimens of endometrial tissue}

Tissue samples were collected from child-bearing-aged women who underwent surgery from February 2019 to January 2020 in the gynecology department of The Second Xiangya Hospital. Fresh proliferation-stage endometriosis cyst samples and endometrial tissue were snap-frozen and stored in a liquid nitrogen container. The study was approved by the ethics committee of The Second Xiangya Hospital. Before the samples were taken, informed consent was assigned by each patient. The clinicopathological data of endometriosis patients, including age, histological subtype, clinical-stage, and cyst size, was retrieved (Supplementary Table 1).
Experimental group: A total of 31 patients with ectopic endometriosis were enrolled, all of whom were diagnosed with ovarian endometriosis by laparoscopy and histopathology. The endometriosis lesions were collected intraoperatively as the ectopic endometrium group (American Fertility Society stage III-IV).

Control group: 10 patients with secondary infertility caused by fallopian tube obstruction factors confirmed by the combined operation of uterus and abdomen. The histological diagnosis is proliferation stage endometrium. The sample size was calculated with equation $\mathrm{N}=\mathrm{Z}^{2} \times(\mathrm{P}$ $\times(1-\mathrm{P})) / \mathrm{E}^{2}$ and based on previous studies [51].

\section{Endometrial and renal cells lines}

Human renal epithelial HEK293T cells and End1/E6E7 endometrial cells from endometriosis patients were purchased from Bena Culture Collection (Beijing, China).

\section{Plasmids for luciferase assay}

pmiR-RB-Report ${ }^{T M}$ hsa_circ_0063526 (hsa_circ_0063 526 3'UTR:1044-1103) -WT (hsa_circ 0063526-WT), pmiR-RB-Report ${ }^{\mathrm{TM}}$ hsa_circ_0063526 (hsa_circ_00635 263'UTR:1044-1103) - MŪT (1273-1280GGAAGA T $>$ CCTTCTA) (hsa_circ_0063526-MUT) plasmids were provided by Ribobio (Guangzhou, China).

\section{Primers used for RT-qPCR detection}

All primers were provided by Ribobio (Guangzhou, China) and verified by NIH BLAST (Bethesda, MD). The primers used in the experiment are shown in Table 1.

\section{Design and synthesis of si-hsa_circ_0063526 and miR- 141-5p inhibitor}

For the sequence of the target gene hsa circ 0063526, siRNA sequence and random independent short RNA sequence (NC-RNA) as the control were designed and synthesized by Genepharma (Suzhou, China): sihsa_circ_0063526-1 Sense: 5 '- GACCCUAACACUGG GUCUGTT-3' Antisense: 5 '- CAGACCCAGUGUUA GGGUCTT-3' si-hsa_circ_0063526-2 Sense: 5 '- AAC

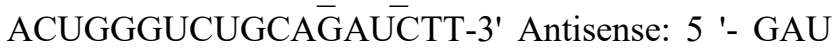
CUGCAGACCCAGUGUUTT-3' si-hsa circ 006352 6-3 Sense: 5 '-CACUGGGUCUGCAGĀUCŪCTT-3' Antisense: 5 '-GAGAUCUGCAGACCCAGUGTT-3' Inhibitor-miR-141-5p: a short-stranded RNA modified by the complementary sequence of miR-141-5p, provided by Ribobio(Guangzhou, China). MicroRNA141-5p sequence is as follows: microRNA-141-5p: 5'UAACACCUGUCUGGUAAAGAUGG-3'.

\section{RNA extraction and RT-qPCR}

RNA was extracted by Trizol reagent (Beijing Dingguo Biological Technology Co., Ltd.). Total RNA was 
Table 1. Primer information used in this paper.

\begin{tabular}{lc}
\hline Primers & Sequences of 5 'to 3' \\
\hline ZO-1 Forward & GAATGATGGTTGGTAGGTGCG \\
ZO-1 Reverse & TCAGAAGTGTGTCTACTGTCCG \\
E-cadherin Forward & TCCATTTCTTGGTCTACGCC \\
E-cadherin Reverse & CACCTTCAGCCAACCTGTTT \\
N-cadherin Forward & GTGCCATTAGCCAAGGGAATTCAGC \\
N-cadherin Reverse & GCGTTCCTGTTCCACTCATAGGAGG \\
Vimentin Forward & AGCCGAAAACACCCTGCAAT \\
Vimentin Reverse & CGTTCAAGGTCAAGACGTGC \\
K-ras Forward & AGACACAAAACAGGCTCAGGA \\
K-ras Reverse & TTCACACAGCCAGGAGTCTTT \\
Beta-actin Forward & GGGGTGTTGAAGGTCTCAAA \\
Beta-actin Reverse & GGCATCCTCACCCTGAAGTA \\
ER-alpha Forward & AAGAGCTGCCAGGCCTGCC \\
ER-alpha Reverse & TTGGCAGCTCTCATGTCTCC \\
ER-beta Forward & GCTCAATTCCAGTATGTACC \\
ER-beta Reverse & GGACCACATTTTTGCACT \\
IL-6 Forward & GTCAACTCCATCTGCCCTTCAG \\
IL-6 Reverse & GGTCTGTTGTGGGTGGTATCCT \\
ZEB1 Forward & TGAATCATCGCTACTCCTACTGT \\
ZEB1 Reverse & TTTCACTGTCTTCATCCTCTTCCC \\
Notch-1 Forward & GTCAACGCCGTAGATGACC \\
Notch-1 Reverse & GTCAACGCCGTAGATGACC \\
MAPK14 Forward & GAACAAGACAATCTGGGAGGTG \\
MAPK14 Reverse & TTCGCATGAATGATGGACTGAA \\
GAPDH Forward & GCACCGTCAAGGCTGAGAAC \\
GAPDH Reverse & TGGTGAAGACGCCAGTGGA \\
hsa_circ_0063526 Forward & AGATTCTGGACCCTAACACTGG \\
hsa_circ_0063526 Reverse & CTCTTGCCTTTGAAACTCAGCT \\
miR-141-5p Forward & CGCGCATCTTCCAGTACAGT \\
miR-141-5p Reverse & AGTGCAGGGTCCGAGGTATT \\
miR - 141-5p reverse transcription & GTCGTATCCAGTGCAGGGTCCGAGGTA- \\
\hline & TTCGCACTGGATACGACTCCAAC \\
\hline
\end{tabular}

reverse transcribed into cDNA, and real-time-qPCR analysis was performed using Universal SYBR Green MasterMix Kit (Vazyme, China). The $2^{-\Delta \Delta \mathrm{Ct}}$ method was used for calculating the relative RNA expression levels [52].

\section{Bioinformatics analysis of hsa_circ_0063526 complementary miRNAs}

Miranda, TargetScan, RNA22 v2, and RNAhybrid software were used to predict the potential target miRNAs and the target genes of the miRNAs involved. The complementary miRNA to hsa_circ_0063526 was predicted, and further research and verification were conducted.

\section{Dual-luciferase reporter assays for circRNA and miRNA binding}

The recombinant plasmid of double luciferase reporter hsa_circ_0063526-WT and hsa_circ_0063526-MUT was designed and synthesized according to the complementary pairing sequence of miR-141-5p and hsa_circ_0063526-MUT. 293T cells were seeded with $1.0 \times 10^{4}$ cells per well. The miRNA mimics or nontarget control vector and target gene 3 'UTR double reporter vector or mutant vector were diluted in $5 \mu \mathrm{L}$ Opti-MEM medium, and the transfection reagent was diluted with $0.25 \mu \mathrm{L}$ Lipo6000 in $5 \mu \mathrm{L}$ Opti-MEm medium. Before plasmids and mimics were added to the cells, $90 \mu \mathrm{L}$ culture medium was added to each well. 
Mimics transfection concentration was $50 \mathrm{nM}$, and plasmid concentration was 50ng/well. Each group included three replicates. After $48 \mathrm{~h}$ of transfection, the medium was extracted and PBS added. Luciferase reagent was added and shaken for $10 \mathrm{~min}$. The medium was transferred to LUMITRACTM 20096 well white cell culture plate for fluorescence determination. Finally, $30 \mu \mathrm{L}$ stop reagent was added to each well for spectrophotometric determination.

In section 2.5-2.8, End1/E6E7 cells were divided into five groups, specifically:

1) si-hsa_circ_0063526 group (si-hsa_circ_0063526 transfection group): The si-hsa_circ_0063526 siRNA was transfected to knockdown hsa_circ_0063526.

2) si-hsa_circ_0063526 + inhibitor-miR-141-5p group (si-hsa_circ_0063526 + inhibitor-miR-141-5p group): the si-hsa circ 0063526 and miR-141-5p were transfected, knockdown of hsa_circ_0063526 siRNA and inhibition of miRNA-141-5p performed at the same time.

3) Inhibitor of miR-141-5p group (inhibitor of miR141-5p transfection group): Transfected the inhibitor of miR-141-5p to inhibit the function of miR-141-5p.

4) NC-RNA group (NC-RNA transfection group): group transfected with random short RNA sequences.

5) Blank control group: transfection reagent and RNA were not added.

\section{Wound healing assays for cell migration}

To detect the migration of cells, we conducted wound healing assays in vitro. Sterile $100 \mu \mathrm{L}$ tips were dragged over the confluent monolayer cells in each cell culture wells to create a gap in the monolayer. Cells were washed with PBS and placed back into the incubator for 48 hours. The cells were observed under an inverted microscope. The width of the scratch was measured and recorded by ImageJ software (NIH, USA).

\section{Transwell invasion assays for cell invasiveness}

To detect the invasion ability of each group, a Transwell assay was performed in vitro. Matrigel was transferred from $-20^{\circ} \mathrm{C}$ to $4^{\circ} \mathrm{C}$ and thawed for 12 hours. The Matrigel was diluted without serum. The Matrigel was carefully placed into an upper chamber with a diameter of $8 \mu \mathrm{m}$. The Transwell plate was placed back into the $\mathrm{CO}_{2}$ incubator at $37^{\circ} \mathrm{C}$ and incubated for 24 hours. $1 \times 10^{4}$ of End1/E6E7 cells in $200 \mu \mathrm{L}$ serum-free cell suspension was added to the upper chamber. $600 \mu \mathrm{L}$ high-sugar DMEM medium containing $10 \%$ fetal bovine serum was added into the lower chamber, and the plate was put back into $\mathrm{CO}_{2}$ at $37^{\circ} \mathrm{C}$ and incubated for 36 hours. The cells were stained with $0.5 \%$ crystal violet and migrated cells were counted with ImageJ software.

\section{EdU assays for cell proliferation}

EdU (5-acetylene 2' -deoxyuridine) proliferation assay was performed using the EdU Apollo567 proliferation in vitro kit (RiboBio, Guangzhou, China).

End1/E6E7 cells $\left(2 \times 10^{4}\right)$ were seeded to a 96-well plate. The cells were transfected with sihsa_circ_0063526, miR-141-5p inhibitor, sicirc $0063526+\mathrm{miR}-141-5 \mathrm{p}$ inhibitor, and control siRNA. After 48 hours, $100 \mu \mathrm{L}$ diluted $50 \mathrm{M}$ EdU was added to each 96-well plate. The cells were then fixed and dyed with $1 \mathrm{X}$ Apollo staining solution and DAPI. The number of EdU positive cells and the total number of cells were calculated immediately, and results were analyzed with ImageJ software.

\section{Enzyme-linked immunosorbent assay (ELISA) for E-cadherin}

Human E-Cadherin ELISA kit (R\&D Systems, MN, USA) was used for E-Cadherin expression detection. $100 \mu \mathrm{L}$ cell culture supernatants of the five groups were first added to the wells of the 96-well ELISA plate, along with a standard curve. The standard curve was set up in concentrations of $0,0.31,0.63,1.25,2.50,5.00$, $10.00,20.00 \mathrm{ng} / \mathrm{ml}$. The enzyme-labeled plates were incubated on a micro-oscillator with a speed of 700rpm for $2 \mathrm{~h}$ and washed 4 times. Next, $200 \mu \mathrm{L}$ of the enzyme marker E-cadherin conjugate was added into each well. Finally, substrate solution and termination solution were added to each well and the OD value at $450 \mathrm{~nm}$ was measured. Using the OD value of E-cadherin standard as ordinate (taking the average value of the concentration of three pores) and dilution concentration of a standard substance as abscissa, the standard curve was drawn, and the E-cadherin contents of the samples were calculated according to the standard curve.

\section{Induction of endometriosis in mice}

The endometrium was obtained from 18 childbearingaged women who underwent hysterectomy for uterine myoma at The Second Xiangya Hospital. Pelvic cavities were examined for the absence of endometriosis during the surgery. According to Noyes et al. criteria (1975), the menstrual cycle has been histologically confirmed as the proliferative phase. The patients had not received hormone therapy for at least six months. All experimental protocols were approved by The Ethics 
Committee under the declaration of Helsinki, and all women signed informed consent. The endometrium was obtained by an endometrial specimen collector (J-ES090500; Cook, USA), cut into identical $2 \mathrm{~mm}$ diameter fragments, and incubated in DMEM, PEN/STREP (Changsha, China) culture medium at $4^{\circ} \mathrm{C}$ before implantation. Female nude mice aged 5 to 6 weeks were purchased and reared in the Department of Laboratory Animals in Central South University. Five animals were maintained in each cage during a 12-hour light and 12hour dark cycle ( 8 a.m. to 8 p.m.) in a barrier system.

The modified endometriosis model previously used in our laboratory and widely by previous scholars was used to induce endometriosis in 30 mice [51]. In this protocol, endometrial tissue fragments of patients of the same size $(2 \mathrm{~mm})$ were sutured to the peritoneal surface of each mice. Mice were anesthetized by sodium pentobarbital and laparotomies were performed through a midline incision. One piece of endometrial tissue fragments was sutured by 5-0 polyglactin suture on the surface of the left and right abdominal wall respectively before the peritoneum and skin were closed.

\section{microRNA-141-5p-agomir and si-hsa_circ_0063526 agomir treatment}

Thirty experimental endometriosis models were randomly allocated into six groups of 5 in each. Five days after induction of endometriosis, miRNA-141-5p therapy began with miRNA-141-5p-agomir (UAACACUGUCUGGUAAAGAUGG Genepharma Company, China) +vector Entranster-R4000 carrier (Engreen Biosystem, China), or scramble miRNAagomir +vector, or only saline as two control groups. sihsa_circ_0063526 agomir therapy began with siRNAhsa_circ_0063526 agomir (sense: 5 'CACUGGGUCUGCAGAUCUCTT-3' Antisense: 5'-GAGAUCUGCAGACCCAGUGTT-3' Genepharma Company, China) +vector or scramble agomir+vector or only saline as two control group. RNAs were intraperitoneally injected into the mice by transfection vector Entranster-R4000 carrier. An EntransterR4000+RNA mimic mixture was prepared. Each injection of $0.5 \mathrm{~mL} 5 \%$ dextrose mixture included $90 \mu \mathrm{g}$ oligonucleotides and $20 \mu \mathrm{L}$ of transfection vector. The mice were injected intraperitoneally every three days for two weeks.

\section{Evaluation of lesions' volume}

After microRNA-141-5p-agomir and sihsa_circ_0063526-agomir treatment for two weeks, the mice were sacrificed, and the endometriosis lesions were collected from the abdominal cavity of mice. The volume of lesions was calculated by the formula of minimum diameter ${ }^{2} *$ maximum diameter/2 [51]. Left abdominal lesions were preserved in RNA stabilized solution (Qiagen, Germany) for extraction of mRNA, RT-qPCR was used to detect gene expression, and the right abdominal lesions were preserved in $4 \%$ polyformaldehyde solution for immunohistochemical study. Endometriosis was observed under a light microscope after $\mathrm{H} \& \mathrm{E}$ staining in a blinded manner. Image $\mathbf{J}$ was used for the calculation of the lesion area. We import the scale bar in Image J. Then use Selection Brush Tool to calculate the total area of endometrial and stroma part.

\section{Immunohistochemistry for epithelial and mesenchymal markers}

We used 4\% paraformaldehyde to fix lesions and paraffin to embed lesions. Tissue was cut into slices about $5 \mu \mathrm{m}$ thick and fixed on glass slides, then boiled in sodium citrate $(\mathrm{pH}=6)$ solution in high pressure for 15 minutes for antigen retrieval. $10 \%$ goat serum was used for antigen blocking. Slides were incubated at $4^{\circ} \mathrm{C}$ overnight with anti-E-cadherin (1:1500; Proteintech, USA), anti-N-cadherin (1:1500; Proteintech, USA), anti-Vimentin (1:1500; Proteintech, USA) antibodies to determine protein expression. Sections were dyed with DAB (Well-Biology, Changsha, China) and counterstained with hematoxylin (Well-Biology, Changsha, China). Stained section images were taken with OLYMPUS BX63 (OLYMPUS, Japan) and analyzed by Image-pro Plus 7.0.

\section{Statistical analysis}

All statistical analysis was performed in GraphPad Prism software (GraphPad, La Jolla, CA), and all in vitro experiments were carried out three times. First, normality and homogeneity of variance were tested in the data obtained from each group. If the sample mean of the group conformed to the homogeneity of variance and normality after comparison, one-way ANOVA was used, and the multiple comparisons between pairs was tested by the least significant difference (LSD) method. If the variance of the group of data is not uniform, the non-parametric Kruskal-Wallis $\mathrm{H}$ test and $\mathrm{U}$ test were used. Pearson's correlation analysis was used for correlation analysis of numerical variables when two variables are in line with normal distribution, and Spearman correlation analysis was used if the two variables were not normally distributed. The test criterion was set to $\alpha=0.05$.

\section{Availability of data and materials}

All data are available to others with investigator support. 


\section{Abbreviations}

EMs: endometriosis; N-cad: N-cadherin; E-cad: Ecadherin; EMT: Epithelial-mesenchymal transition; ncRNAs: non-coding RNA; circRNA: circular RNA; miRNA: microRNA; RNA: Ribonucleic Acid; ZO-1: Zona occludens 1; PVDF: polyvinylidene difluoride; 3'UTR: 3'- untranslated region; ceRNA: competing endogenous RNA; BS: back splicing; RNAi: RNA interference; siRNA: small interference RNA; EdU: 5acetylene 2' -deoxyuridine; PBS: phosphate buffer saline; VEGF: Vascular endothelial growth factor.

\section{AUTHOR CONTRIBUTIONS}

Zhangming Wei contributed the central idea, analyzed most of the data. Mengmeng Zhang participates in writing the initial draft of the paper. Lipin $\mathrm{Li}$ contributed to refining the ideas, carrying out additional analyses. Xiaoling Fang finalizes the revision of this paper. Xinyue Zhang has verified the underlying data.

\section{ACKNOWLEDGMENTS}

We thank all members of our research group for their time and effort in providing precious feedback and insightful comments.

\section{CONFLICTS OF INTEREST}

The authors declare that they have no conflicts of interest.

\section{FUNDING}

This study was funded by The National Natural Science Foundation of China (81671437, 81771558) and Shenzhen People's Hospital. The authors thank AiMi Academic Services (www.aimieditor.com) for English language editing and review services.

\section{REFERENCES}

1. Escudero-Lara A, Argerich J, Cabañero D, Maldonado R. Disease-modifying effects of natural $\Delta 9$ tetrahydrocannabinol in endometriosis-associated pain. Elife. 2020; 9:e50356.

https://doi.org/10.7554/eLife.50356

PMID:31931958

2. Hickey M, Ballard K, Farquhar C. Endometriosis. BMJ. 2014; 348:g1752.

https://doi.org/10.1136/bmj.g1752

PMID:24647161

3. Koninckx PR, Ussia A, Adamyan L, Wattiez A, Gomel V, Martin DC. Pathogenesis of endometriosis: the genetic/epigenetic theory. Fertil Steril. 2019; 111:327-40.

https://doi.org/10.1016/j.fertnstert.2018.10.013

PMID:30527836

4. Wei Z, Zhang M, Zhang X, Yi M, Xia X, Fang X. NAT2 gene polymorphisms and endometriosis risk: $A$ PRISMA-compliant meta-analysis. PLoS One. 2019; 14:e0227043.

https://doi.org/10.1371/journal.pone.0227043 PMID:31881062

5. Vercellini $P$, Viganò $P$, Somigliana $E$, Fedele $L$. Endometriosis: pathogenesis and treatment. Nat Rev Endocrinol. 2014; 10:261-75. https://doi.org/10.1038/nrendo.2013.255 PMID:24366116

6. Symons LK, Miller JE, Kay VR, Marks RM, Liblik K, Koti M, Tayade C. The Immunopathophysiology of Endometriosis. Trends Mol Med. 2018; 24:748-62. https://doi.org/10.1016/j.molmed.2018.07.004 PMID:

7. Brown J, Farquhar C. An overview of treatments for endometriosis. JAMA. 2015; 313:296-7.

https://doi.org/10.1001/jama.2014.17119 PMID:25603001

8. Barbieri RL. New therapy for endometriosis. N Engl J Med. 1988; 318:512-4. https://doi.org/10.1056/NEJM198802253180810 PMID:2963214

9. Berkley KJ, Rapkin AJ, Papka RE. The pains of endometriosis. Science. 2005; 308:1587-9. https://doi.org/10.1126/science.1111445 PMID:15947176

10. Diamond MP, DeCherney AH. Nafarelin for treatment of endometriosis. N Engl J Med. 1988; 319:519-20. https://doi.org/10.1056/NEJM198808253190814 PMID: 2970010

11. Adammek M, Greve B, Kässens N, Schneider C, Brüggemann K, Schüring AN, Starzinski-Powitz A, Kiesel L, Götte M. MicroRNA miR-145 inhibits proliferation, invasiveness, and stem cell phenotype of an in vitro endometriosis model by targeting multiple cytoskeletal elements and pluripotency factors. Fertil Steril. 2013; 99:1346-55.e5.

https://doi.org/10.1016/i.fertnstert.2012.11.055 PMID:23312222

12. Park JH, Lee SK, Kim MK, Lee JH, Yun BH, Park JH, Seo SK, Cho S, Choi YS. Saponin Extracts Induced Apoptosis of Endometrial Cells From Women With Endometriosis Through Modulation of miR-21-5p. Reprod Sci. 2018; 25:292-301.

https://doi.org/10.1177/1933719117711263 PMID:28558522 
13. Pei T, Liu C, Liu T, Xiao L, Luo B, Tan J, Li X, Zhou G, Duan C, Huang W. miR-194-3p Represses the Progesterone Receptor and Decidualization in Eutopic Endometrium From Women With Endometriosis. Endocrinology. 2018; 159:2554-62.

https://doi.org/10.1210/en.2018-00374 PMID:29762665

14. Rekker $K$, Saare $M$, Roost AM, Kaart T, Sõritsa $D$, Karro H, Sõritsa A, Simón C, Salumets A, Peters M. Circulating miR-200-family micro-RNAs have altered plasma levels in patients with endometriosis and vary with blood collection time. Fertil Steril. 2015; 104:938-46.e2.

https://doi.org/10.1016/j.fertnstert.2015.06.029 PMID:26206343

15. Rekker K, Tasa T, Saare M, Samuel K, Kadastik Ü, Karro H, Götte M, Salumets A, Peters M. DifferentiallyExpressed miRNAs in Ectopic Stromal Cells Contribute to Endometriosis Development: The Plausible Role of miR-139-5p and miR-375. Int J Mol Sci. 2018; 19:3789. https://doi.org/10.3390/ijms19123789

PMID:30487429

16. Maged AM, Deeb WS, El Amir A, Zaki SS, El Sawah H, Al Mohamady M, Metwally AA, Katta MA. Diagnostic accuracy of serum miR-122 and miR-199a in women with endometriosis. Int J Gynaecol Obstet. 2018; 141:14-9.

https://doi.org/10.1002/ijgo.12392 PMID:29149541

17. Yang $P$, Wu Z, Ma C, Pan N, Wang Y, Yan L. Endometrial miR-543 Is Downregulated During the Implantation Window in Women With Endometriosis-Related Infertility. Reprod Sci. 2019; 26:900-8.

https://doi.org/10.1177/1933719118799199

PMID:30231774

18. Wu D, Lu P, Mi X, Miao J. Exosomal miR-214 from endometrial stromal cells inhibits endometriosis fibrosis. Mol Hum Reprod. 2018; 24:357-65.

https://doi.org/10.1093/molehr/gay019

PMID:29660008

19. Yang WW, Hong $L, X u X X$, Wang $Q$, Huang JL, Jiang $L$. Regulation of miR-33b on endometriosis and expression of related factors. Eur Rev Med Pharmacol Sci. 2017; 21:2027-33.

PMID:28537685

20. Wang L, Huang W, Ren C, Zhao M, Jiang X, Fang X, Xia $X$. Analysis of Serum microRNA Profile by Solexa Sequencing in Women With Endometriosis. Reprod Sci. 2016; 23:1359-70. https://doi.org/10.1177/1933719116641761 PMID:27412772

21. Kim MK, Moon YA, Song CK, Baskaran R, Bae S, Yang SG. Tumor-suppressing miR-141 gene complex- loaded tissue-adhesive glue for the locoregional treatment of hepatocellular carcinoma. Theranostics. 2018; 8:3891-901.

https://doi.org/10.7150/thno.24056 PMID:30083268

22. Liang Z, Chen $Y$, Zhao Y, Xu C, Zhang A, Zhang Q, Wang D, He J, Hua W, Duan P. miR-200c suppresses endometriosis by targeting MALAT1 in vitro and in vivo. Stem Cell Res Ther. 2017; 8:251. https://doi.org/10.1186/s13287-017-0706-z PMID:29116025

23. Zhang $M$, Wang $S$, Tang $L$, Wang $X$, Zhang $T$, Xia X, Fang X. Downregulated circular RNA hsa_circ_0067301 regulates epithelial-mesenchymal transition in endometriosis via the miR-141/Notch signaling pathway. Biochem Biophys Res Commun. 2019; 514:71-7.

https://doi.org/10.1016/j.bbrc.2019.04.109 PMID:31023528

24. Meng S, Zhou H, Feng Z, Xu Z, Tang Y, Li P, Wu M. CircRNA: functions and properties of a novel potential biomarker for cancer. Mol Cancer. 2017; 16:94.

https://doi.org/10.1186/s12943-017-0663-2 PMID:28535767

25. Hollensen $A K$, Thomsen $H S$, Lloret-Llinares $M$, Kamstrup $A B$, Jensen JM, Luckmann $M$, Birkmose $N$, Palmfeldt J, Jensen TH, Hansen TB, Damgaard CK. circZNF827 nucleates a transcription inhibitory complex to balance neuronal differentiation. Elife. 2020; 9:e58478.

https://doi.org/10.7554/eLife.58478 PMID:33174841

26. Pei $C$, Wang $H$, Shi C, Zhang C, Wang M. CircRNA hsa_circ_0013958 may contribute to the development of ovarian cancer by affecting epithelial-mesenchymal transition and apoptotic signaling pathways. J Clin Lab Anal. 2020; 34:e23292.

https://doi.org/10.1002/jcla.23292 PMID:32157748

27. Hu J, Wang L, Chen J, Gao H, Zhao W, Huang Y, Jiang T, Zhou J, Chen Y. The circular RNA circ-ITCH suppresses ovarian carcinoma progression through targeting miR145/RASA1 signaling. Biochem Biophys Res Commun. 2018; 505:222-8.

https://doi.org/10.1016/j.bbrc.2018.09.060

PMID: $\underline{30243714}$

28. Lin W, Ye H, You K, Chen L. Up-regulation of circ_LARP4 suppresses cell proliferation and migration in ovarian cancer by regulating miR-513b-5p/LARP4 axis. Cancer Cell Int. 2020; 20:5.

https://doi.org/10.1186/s12935-019-1071-z PMID:31911757

29. Luo L, Gao Y, Sun X. Circ-ITCH correlates with small tumor size, decreased FIGO stage and prolonged overall survival, and it inhibits cells proliferation while 
promotes cells apoptosis in epithelial ovarian cancer. Cancer Biomark. 2018; 23:505-13.

https://doi.org/10.3233/CBM-181609 PMID:30347599

30. Li L, Yu P, Zhang P, Wu H, Chen Q, Li S, Wang Y. Upregulation of hsa_circ_0007874 suppresses the progression of ovarian cancer by regulating the miR-760/SOCS3 pathway. Cancer Med. 2020; 9:2491-9.

https://doi.org/10.1002/cam4.2866 PMID:32023009

31. Xu X, Jia SZ, Dai Y, Zhang JJ, Li X, Shi J, Leng J, Lang J. The Relationship of Circular RNAs With Ovarian Endometriosis. Reprod Sci. 2018; 25:1292-1300.

https://doi.org/10.1177/1933719118759439 PMID:29490568

32. Zhang $M$, Ren $C$, Xiao $Y$, Xia $X$, Fang X. Expression Profile Analysis of Circular RNAs in Ovarian Endometriosis by Microarray and Bioinformatics. Med Sci Monit. 2018; 24:9240-50.

https://doi.org/10.12659/MSM.913885 PMID:30566420

33. Shen $L$, Zhang $Y$, Zhou $W$, Peng $Z$, Hong $X$, Zhang $Y$. Circular RNA expression in ovarian endometriosis. Epigenomics. 2018; 10:559-72.

https://doi.org/10.2217/epi-2017-0079

PMID:29334789

34. Wang D, Luo Y, Wang G, Yang Q. Circular RNA expression profiles and bioinformatics analysis in ovarian endometriosis. Mol Genet Genomic Med. 2019; 7:e00756.

https://doi.org/10.1002/mgg3.756

PMID:31144476

35. Chen Q, Zhang J, He Y, Wang Y. hsa_circ_0061140 Knockdown Reverses FOXM1-Mediated Cell Growth and Metastasis in Ovarian Cancer through miR-370 Sponge Activity. Mol Ther Nucleic Acids. 2018; 13:55-63.

https://doi.org/10.1016/i.omtn.2018.08.010

PMID:30236833

36. Anglesio MS, Papadopoulos N, Ayhan A, Nazeran TM, Noë M, Horlings HM, Lum A, Jones S, Senz J, Seckin T, Ho J, Wu RC, Lac V, et al. Cancer-Associated Mutations in Endometriosis without Cancer. N Engl J Med. 2017; 376:1835-48.

https://doi.org/10.1056/NEJMoa1614814 PMID:28489996

37. Bartley J, Jülicher A, Hotz B, Mechsner S, Hotz H. Epithelial to mesenchymal transition (EMT) seems to be regulated differently in endometriosis and the endometrium. Arch Gynecol Obstet. 2014; 289:871-81.

https://doi.org/10.1007/s00404-013-3040-4

PMID:24170160
38. Henkel A, Christensen B, Schindler AE. Endometriosis: a clinically malignant disease. Eur J Obstet Gynecol Reprod Biol. 1999; 82:209-11.

https://doi.org/10.1016/s0301-2115(98)00249-8 PMID:10206417

39. Konrad L, Dietze R, Riaz MA, Scheiner-Bobis G, Behnke J, Horné F, Hoerscher A, Reising C, Meinhold-Heerlein I. Epithelial-Mesenchymal Transition in EndometriosisWhen Does It Happen? J Clin Med. 2020; 9:1915. https://doi.org/10.3390/jcm9061915 PMID:32570986

40. Matsuzaki S, Darcha C. Epithelial to mesenchymal transition-like and mesenchymal to epithelial transition-like processes might be involved in the pathogenesis of pelvic endometriosis. Hum Reprod. 2012; 27:712-21.

https://doi.org/10.1093/humrep/der442 PMID:22215621

41. Albertsen HM, Ward K. Genes Linked to Endometriosis by GWAS Are Integral to Cytoskeleton Regulation and Suggests That Mesothelial Barrier Homeostasis Is a Factor in the Pathogenesis of Endometriosis. Reprod Sci. 2017; 24:803-11. https://doi.org/10.1177/1933719116660847 PMID:27470151

42. Du $\mathrm{Y}$, Zhang $\mathrm{Z}$, Xiong $\mathrm{W}$, Li $\mathrm{N}$, Liu $\mathrm{H}, \mathrm{He} \mathrm{H}$, Li Q, Liu $\mathrm{Y}$, Zhang L. Estradiol promotes EMT in endometriosis via MALAT1/miR200s sponge function. Reproduction. 2019; 157:179-88.

https://doi.org/10.1530/REP-18-0424

PMID:30500775

43. Saha $R$, Pettersson $H J$, Svedberg $P$, Olovsson $M$, Bergqvist A, Marions L, Tornvall P, Kuja-Halkola R. Heritability of endometriosis. Fertil Steril. 2015; 104:947-52.

https://doi.org/10.1016/j.fertnstert.2015.06.035 PMID:26209831

44. Mashayekhi P, Noruzinia M, Zeinali S, Khodaverdi S. Endometriotic Mesenchymal Stem Cells Epigenetic Pathogenesis: Deregulation of miR-200b, miR-145, and let7b in A Functional Imbalanced Epigenetic Disease. Cell J. 2019; 21:179-85. https://doi.org/10.22074/cellj.2019.5903 PMID:30825291

45. Tajan M, Paccoud R, Branka S, Edouard T, Yart A. The RASopathy Family: Consequences of Germline Activation of the RAS/MAPK Pathway. Endocr Rev. 2018; 39:676-700. https://doi.org/10.1210/er.2017-00232 PMID:29924299

46. Chatterjee K, Jana S, DasMahapatra P, Swarnakar S. EGFR-mediated matrix metalloproteinase-7 upregulation promotes epithelial-mesenchymal transition 
via ERK1-AP1 axis during ovarian endometriosis progression. FASEB J. 2018; 32:4560-72.

https://doi.org/10.1096/fj.201701382RR

PMID:29558202

47. Zhang $\mathrm{M}, \mathrm{Xia} \mathrm{B}, \mathrm{Xu} \mathrm{Y}$, Zhang Y, Xu J, Lou G. Circular RNA (hsa_circ_0051240) promotes cell proliferation, migration and invasion in ovarian cancer through miR637/KLK4 axis. Artif Cells Nanomed Biotechnol. 2019; 47:1224-33.

https://doi.org/10.1080/21691401.2019.1593999

PMID:30945557

48. Zhao Y, Qin XP, Lang YP, Kou D, Shao ZW. Circular RNA circ-SMAD7 promoted ovarian cancer cell proliferation and metastasis by suppressing KLF6. Eur Rev Med Pharmacol Sci. 2019; 23:5603-10.

https://doi.org/10.26355/eurrev 20190718294 PMID:31298312

49. Panir K, Schjenken JE, Robertson SA, Hull ML. Noncoding RNAs in endometriosis: a narrative review. Hum Reprod Update. 2018; 24:497-515. https://doi.org/10.1093/humupd/dmy014

PMID:29697794

50. Liu CH, Sun Y, Li J, Gong Y, Tian KT, Evans LP, Morss PC, Fredrick TW, Saba NJ, Chen J. Endothelial microRNA150 is an intrinsic suppressor of pathologic ocular neovascularization. Proc Natl Acad Sci USA. 2015; 112:12163-8.

https://doi.org/10.1073/pnas.1508426112

PMID:26374840

51. Sahin C, Mamillapalli R, Yi KW, Taylor HS. microRNA Let-7b: A Novel treatment for endometriosis. J Cell Mol Med. 2018; 22:5346-53.

https://doi.org/10.1111/jcmm.13807

PMID:30063121

52. Rao X, Huang X, Zhou Z, Lin X. An improvement of the $2^{\wedge}$ (-delta delta CT) method for quantitative real-time polymerase chain reaction data analysis. Biostat Bioinforma Biomath. 2013; 3:71-85.

PMID:25558171 


\section{SUPPLEMENTARY MATERIALS}

\section{Supplementary Figure}

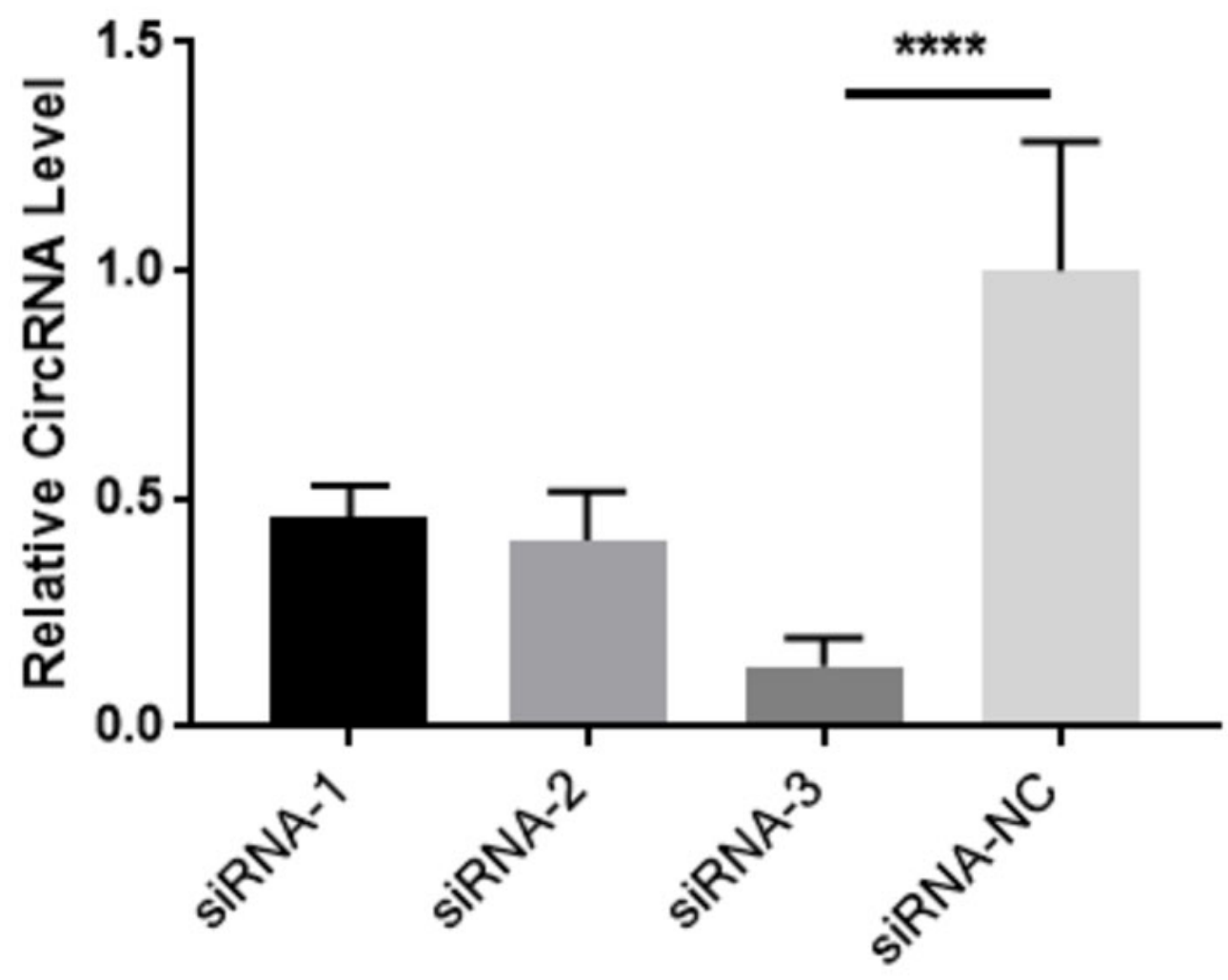

Supplementary Figure 1. Relative expression level of hsa_circ0063526 in End1/E6E7 cells after siRNA transfection. Replicated 3 times involving 3 samples $(* * * * P<0.001)$. 
Supplementary Table

Supplementary Table 1. Clinical characteristics of the patients.

\begin{tabular}{|c|c|c|c|c|c|}
\hline Specimen number & Diseases & The menstrual cycle & Source of parts & Age & Stage \\
\hline YW-B-01 & Ovarian endometriosis & Proliferative phase & The right ovary & 28 & IV \\
\hline YW-B-02 & Ovarian endometriosis & Proliferative phase & The right ovary & 30 & III \\
\hline YW-B-03 & Ovarian endometriosis & Proliferative phase & The right ovary & 41 & III \\
\hline YW-B-04 & Ovarian endometriosis & Proliferative phase & The right ovary & 26 & III \\
\hline YW-B-05 & Ovarian endometriosis & Proliferative phase & The right ovary & 26 & III \\
\hline YW-B-06 & Ovarian endometriosis & Proliferative phase & The left ovary & 31 & IV \\
\hline YW-B-07 & Ovarian endometriosis & Proliferative phase & The left ovary & 28 & IV \\
\hline YW-B-08 & Ovarian endometriosis & Proliferative phase & The left ovary & 28 & IV \\
\hline YW-B-09 & Ovarian endometriosis & Proliferative phase & The right ovary & 38 & III \\
\hline YW-B-10 & Ovarian endometriosis & Proliferative phase & The right ovary & 24 & III \\
\hline YW-B-11 & Ovarian endometriosis & Proliferative phase & The right ovary & 28 & III \\
\hline YW-B-12 & Ovarian endometriosis & Proliferative phase & The right ovary & 29 & III \\
\hline YW-B-13 & Ovarian endometriosis & Proliferative phase & The right ovary & 26 & III \\
\hline YW-B-14 & Ovarian endometriosis & Proliferative phase & The left ovary & 38 & IV \\
\hline YW-B-15 & Ovarian endometriosis & Proliferative phase & The right ovary & 34 & III \\
\hline YW-B-16 & Ovarian endometriosis & Proliferative phase & The left ovary & 35 & IV \\
\hline YW-B-17 & Ovarian endometriosis & Proliferative phase & The left ovary & 31 & IV \\
\hline YW-B-18 & Ovarian endometriosis & Proliferative phase & The left ovary & 29 & III \\
\hline YW-B-19 & Ovarian endometriosis & Proliferative phase & The right ovary & 27 & III \\
\hline YW-B-20 & Ovarian endometriosis & Proliferative phase & The right ovary & 38 & IV \\
\hline YW-B-21 & Ovarian endometriosis & Proliferative phase & Bilateral ovaries & 26 & IV \\
\hline YW-B-22 & Ovarian endometriosis & Proliferative phase & The left ovary & 35 & IV \\
\hline YW-B-23 & Ovarian endometriosis & Proliferative phase & The left ovary & 30 & III \\
\hline YW-B-24 & Ovarian endometriosis & Proliferative phase & The right ovary & 38 & IV \\
\hline YW-B-25 & Ovarian endometriosis & Proliferative phase & The right ovary & 38 & III \\
\hline YW-B-26 & Ovarian endometriosis & Proliferative phase & The right ovary & 29 & III \\
\hline YW-B-27 & Ovarian endometriosis & Proliferative phase & The right ovary & 31 & III \\
\hline YW-B-28 & Ovarian endometriosis & Proliferative phase & The right ovary & 29 & III \\
\hline YW-B-29 & Ovarian endometriosis & Proliferative phase & The right ovary & 38 & III \\
\hline YW-B-30 & Ovarian endometriosis & Proliferative phase & The right ovary & 27 & III \\
\hline YW-B-31 & Ovarian endometriosis & Proliferative phase & The right ovary & 26 & IV \\
\hline DZ-N-01 & Secondary infertility & Proliferative phase & Endometrium & 40 & - \\
\hline DZ-N-02 & Secondary infertility & Proliferative phase & Endometrium & 41 & - \\
\hline DZ-N-03 & Secondary infertility & Proliferative phase & Endometrium & 32 & - \\
\hline DZ-N-04 & Secondary infertility & Proliferative phase & Endometrium & 30 & - \\
\hline DZ-N-05 & Secondary infertility & Proliferative phase & Endometrium & 27 & - \\
\hline DZ-N-06 & Secondary infertility & Proliferative phase & Endometrium & 26 & - \\
\hline DZ-N-07 & Secondary infertility & Proliferative phase & Endometrium & 39 & - \\
\hline DZ-N-08 & Secondary infertility & Proliferative phase & Endometrium & 29 & - \\
\hline DZ-N-09 & Secondary infertility & Proliferative phase & Endometrium & 43 & - \\
\hline DZ-N-10 & Secondary infertility & Proliferative phase & Endometrium & 27 & - \\
\hline
\end{tabular}

